\title{
De Finettian Logics of Indicative Conditionals Part II: Proof Theory and Algebraic Semantics
}

\author{
Paul Égré ${ }^{1}$ (1) . Lorenzo Rossi ${ }^{2}$ (i) . Jan Sprenger ${ }^{3}$ (I)
}

Received: 12 November 2019 / Published online 29 January 2021

(C) The Author(s) 2021

\begin{abstract}
In Part I of this paper, we identified and compared various schemes for trivalent truth conditions for indicative conditionals, most notably the proposals by de Finetti (1936) and Reichenbach $(1935,1944)$ on the one hand, and by Cooper (Inquiry, 11, 295-320, 1968) and Cantwell (Notre Dame Journal of Formal Logic, 49, 245$260,2008)$ on the other. Here we provide the proof theory for the resulting logics DF/TT and CC/TT, using tableau calculi and sequent calculi, and proving soundness and completeness results. Then we turn to the algebraic semantics, where both logics have substantive limitations: DF/TT allows for algebraic completeness, but not for the construction of a canonical model, while CC/TT fails the construction of a Lindenbaum-Tarski algebra. With these results in mind, we draw up the balance and sketch future research projects.
\end{abstract}

Keywords Indicative conditionals - Trivalent logics - Cooper-Cantwell conditional · de Finetti conditional · Proof theory · Algebraic semantics · Connexive logics

\footnotetext{
We express our thanks to an anonymous referee of this journal as well as to the colleagues acknowledged in Part I of this paper. Funding for this research was provided by grants ANR-14-CE30-0010 (program TRILOGMEAN), ANR-17-EURE-0017 (program FRONTCOG), and ANR-19-CE28-0004-01 (program PROBASEM) (P.E.), by the Fonds zur Förderung der wissenschaftlichen Forschung (FWF), grant no. P29716-G24 for research carried out at the University of Salzburg (L.R.), and by the European Research Council (ERC) through Starting Grant No. 640638 (J.S.). The order between the three authors is alphabetical; the authors' contribution is equal in part I; in the present part II, P.E. and J.S. would like to acknowledge L.R.'s preponderant contribution to sections 3 and 4.
}

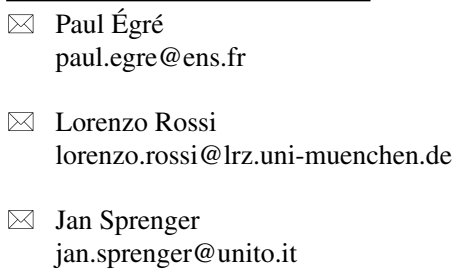

Extended author information available on the last page of the article. 


\begin{tabular}{l|ccccc|ccc}
$f_{\rightarrow_{\mathrm{DF}}}$ & 1 & $1 / 2$ & 0 & & $f_{\rightarrow \mathrm{CC}}$ & 1 & $1 / 2$ & 0 \\
\hline 1 & 1 & $1 / 2$ & 0 & & 1 & 1 & $1 / 2$ & 0 \\
$1 / 2$ & $1 / 2$ & $1 / 2$ & $1 / 2$ & & $1 / 2$ & 1 & $1 / 2$ & 0 \\
0 & $1 / 2$ & $1 / 2$ & $1 / 2$ & & 0 & $1 / 2$ & $1 / 2$ & $1 / 2$
\end{tabular}

Fig. 1 Truth tables for the de Finetti conditional (left) and the Cooper-Cantwell conditional (right)

\section{Introduction}

In Part I of this paper, we reviewed the motivations for a trivalent semantic treatment of indicative conditionals, centered on the proposal made by de Finetti [13] and Reichenbach [36, 37] to treat indicative conditionals as conditional assertions akin to conditional bets. We singled out two de Finettian logics of the indicative conditional, the first based on de Finetti's table, paired with a notion of logical consequence as preservation of non-Falsity (TT-validity), the other based on a close kin to de Finetti's table, the Cooper-Cantwell table, paired with the same notion of validity $[10,12]$. These logics are called DF/TT and CC/TT, respectively. We repeat the truth tables of the conditional operator in Fig. 1 and the definition of TT-validity below.

TT-validity $\quad \Gamma \models \mathrm{x} / \mathrm{TT} A$ provided every $\mathrm{X}$-evaluation that makes all sentences of $\Gamma$ T-true also makes $A$ T-true. In other words, if for every $\mathrm{X}$-evaluation function $v:$ For $\longmapsto\{0,1 / 2,1\}$ (where $\mathrm{X}$ specifies the interpretation of $\rightarrow$ and other connectives), for every sentence $B \in \Gamma, v(B) \in\{1 / 2,1\}$, then also $v(A) \in\{1 / 2,1\}$.

As easily seen from the tables, both conditionals are de Finettian in the following sense: they take the value of the consequent when the antecedent is true $(=1)$, and the value indeterminate when the antecedent is false $(=0)$. They differ when the antecedent itself is indeterminate $(=1 / 2)$ : whereas the DF conditional groups $1 / 2$ with 0 in antecedent position, the CC conditional groups $1 / 2$ with 1 instead. In both logics (whether X=DF or CC), the non-conditional connectives ' $\neg$ ', ' $\wedge$ ' and ' $\vee$ ' are interpreted according to the Łukasiewicz/de Finetti/Strong Kleene truth tables, where negation swaps 1 and 0 , mapping $1 / 2$ to itself, and where conjunction and disjunction are interpretable as min and max respectively. Although alternative tables are given in Cooper [12] for disjunction and conjunction (see Part I of this paper, Section 6), our focus remains on the standard interpretation of the connectives.

In Part I, it was pointed out that the resulting logics DF/TT and CC/TT share some distinctive features. In particular, both satisfy Conditional Introduction and the law of Import-Export, and both are connexive logics, supporting $\neg(A \rightarrow \neg A)$ and the inference from $(A \rightarrow \neg B)$ to $\neg(A \rightarrow B)$. Moreover, both support unrestricted commutation of the conditional with negation. This feature sets them apart from other de Finettian logics, in particular Farrell's [16] and further variants (called de Finettian-Jeffrey in part I, after [22]). ${ }^{1}$ They differ foremost on Modus Ponens, which is preserved in CC/TT but given up in DF/TT.

\footnotetext{
${ }^{1}$ As explained in part I, Farrell's [16] F-variant differs from the Cooper-Cantwell table just on the entry $(1 / 2,1)$, where it returns the value $1 / 2$ : the resulting logic, $F / T T$, is also connexive, but does not support full commutation.
} 
In this second part of our inquiry, we turn to an investigation of the proof theory of DF/TT and CC/TT. We proceed in three main steps: in Section 2, we give sound and complete tableau calculi for either logic; in Section 3, we present sound and complete sequent calculi; in Section 4, finally, we examine the prospect for an algebraic semantics for both DF/TT and CC/TT. As we shall see, neither logic admits a 'nice' algebraic semantics, but there is a sense in which CC/TT, despite satisfying Modus Ponens, falls even shorter than DF/TT in that regard. We give a discussion of that result and compare both logics in Section 5.

\section{Tableau Calculi}

In this section, we introduce sound and complete tableau calculi for CC/TT and DF/TT. Tableau calculi are a proof-theoretical formalism that is very close to the semantics. To prove a sentence, tableaux employ trees that can be conceptualized as reverse truth tables. In building a tableau, one starts from the assumption that certain sentences $A_{0}$, $\ldots, A_{n}$ have certain semantic values, and iteratively works out all the value assignments to the sub-sentences of $A_{0}, \ldots, A_{n}$ that result from the initial assignment. In the propositional case, this process always terminates after a finite number of steps, resulting in either an open or a closed tableau: in the former case, the initial assignment is possible according to the chosen semantics, whereas in the latter it is not. Therefore, in order to prove that $A$ follows from a (finite) set of sentences $\Gamma$ in a tableau system, one shows that all the tableaux resulting from the initial assignments in which all the sentences in $\Gamma$ have a designated value but $A$ does not are closed.

\subsection{Tableau Calculus for CC/TT}

The CC/TT tableau calculus, in symbols CC/TTt, is given by the following tableau construction rules:

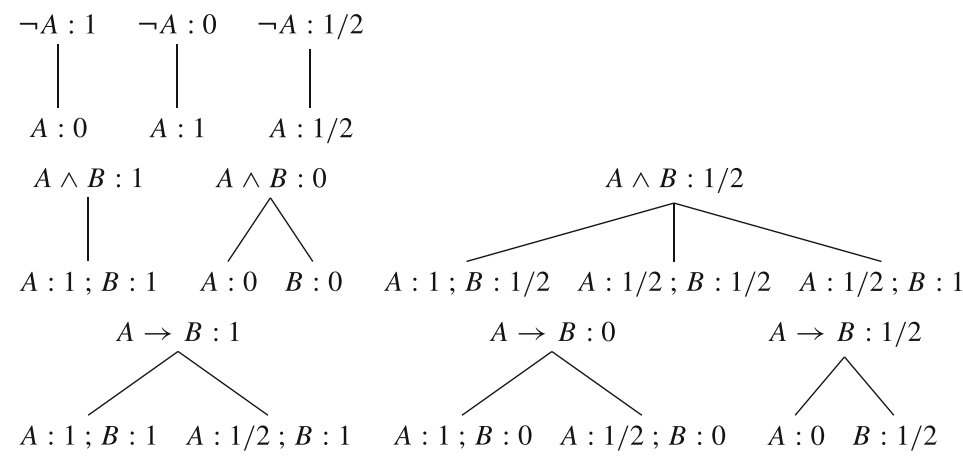

Tableau rules are essentially versions of the truth-table semantics for the target logic, in our case CC/TT. To see this, consider the first rule for the conditional, the one having $A \rightarrow B: 1$ as premise. From this premise, one derives two nodes as consequences, one labelled with $A: 1 ; B: 1$ and one labelled with $A: 1 / 2 ; B: 1$. But these two consequences correspond exactly to the conditions for a conditional $A \rightarrow B$ to have 
value 1 in the truth tables for the Cooper-Cantwell conditional: $A \rightarrow B$ has value 1 in these tables if either both $A$ and $B$ have value 1 , or if $A$ has value $1 / 2$ and $B$ has value 1 (see Fig. 1). Similar considerations apply to the other rules.

We now give a precise characterization of the tableaux generated according to the above rules, and of CC/TTt-derivability.

Definition 2.1 - For every formula $A$, the CC/TTt- $n$-tableau of $A$ (for $n=0,1 / 2$, or 1 ) is the tree whose root is $A: n$, and that is obtained by applying the rules of CC/TTt.

- For every finite set of formulae $\Gamma=\left\{B_{0}, \ldots, B_{k}\right\}$, the CC/TTt- $\left\langle n_{0} ; \ldots ; n_{k}\right\rangle$ tableau of $A$ (for $n_{i}=0,1 / 2$, or 1 , and $i \in\{0, \ldots, k\}$ ) is the tree whose root is $\left\langle B_{0}: n_{0} ; \ldots ; B_{k}: n_{k}\right\rangle$, obtained by applying the rules of CC/TTt. ${ }^{2}$

Since we are only concerned with the tableau calculus for CC/TT in this subsection, we suppress the label 'CC/TTt' whenever possible, to improve readability.

\section{Definition 2.2}

- A branch $\mathcal{B}$ of an $n$-tableau is closed if, for some formula $A$, there are at least two nodes in $\mathcal{B}$ that have $A: m$ and $A: n$ in their labels, and $n \neq m$. A branch is open if it is not closed.

- An $n$-tableau is closed if all its branches are closed, and open otherwise.

Definition 2.3 For every finite set of formulae $\Gamma$ of cardinality $k$ and every formula $A, A$ is CC/TTt-deducible from a $\Gamma$, in symbols $\Gamma \vdash \mathrm{cc} / \mathrm{TTt} A$, if and only if all its $\left\langle n_{0} ; \ldots ; n_{k}\right\rangle$-tableaux are closed, where we use the indices $n_{1}, \ldots, n_{k-1}$ to range over elements in $\{1 / 2,1\}$, and we set $n_{k}=0$.

Before proving soundness and completeness for CC/TTt, we give a sample of how to reason in this calculus. In particular, we prove (one direction of) the commutation with negation in CC/TTt. The following two closed tableaux establish that $A \rightarrow \neg B$ follows from $\neg(A \rightarrow B)$ in CC/TTt. The first tableau shows that $\neg(A \rightarrow B)$ cannot have value 1 while $A \rightarrow \neg B$ has value 0 .

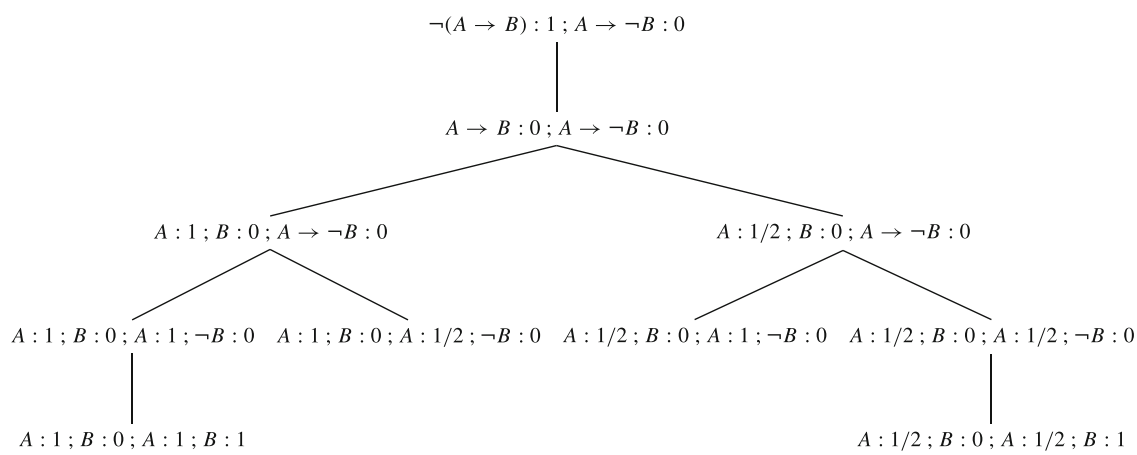

\footnotetext{
${ }^{2}$ To ensure uniqueness in the definition of tableaux for more than one sentence, one should fix a convention for the order in which the CC/TTt rules are applied. Nothing crucial hinges on this, so we don't specify any such convention for the sake of readability.
} 
The second tableau shows that $\neg(A \rightarrow B)$ cannot have value $1 / 2$ while $A \rightarrow \neg B$ has value 0 .

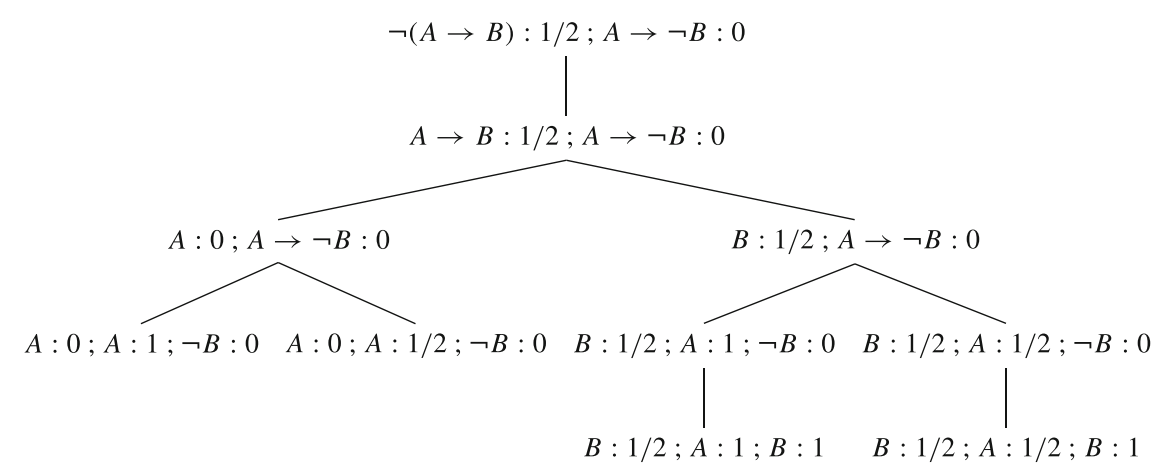

The claim that $\neg(A \rightarrow B)$ follows from $A \rightarrow \neg B$ in CC/TTt is established in a similar fashion.

We now prove that $\mathrm{CC} / \mathrm{TTt}$ is sound and complete with respect to CC/TTvalidity.

Definition 2.4 A quasi-CC-evaluation is a non-total function from the formulae of $\mathcal{L}_{\rightarrow}$ to $\{0,1 / 2,1\}$ that is compatible with the CC truth tables.

More compactly, a quasi-CC-evaluation is a proper subset of a CC-evaluation. For example, the function that sends $p$ and $(p \wedge q)$ to 1 is a quasi- CC-evaluation.

Lemma 2.5 For every finite set $\left\{B_{1}, \ldots, B_{k}\right\}$ of formulae and every CC-evaluation $v$, the completed $\mathrm{CC} / \mathrm{TTt}$-tableau whose root is

$$
B_{1}: v\left(B_{1}\right) ; \ldots ; B_{k}: v\left(B_{k}\right)
$$

is open, and all partial functions from sentences to $\{1,1 / 2,0\}$ induced by its open branches are quasi-CC-evaluations.

Proof By induction on the height of the tree.

- The tableau consisting only of the root $B_{1}: v\left(B_{1}\right) ; \ldots ; B_{k}: v\left(B_{k}\right)$ is open. For suppose it is closed. Then, there are at least two sentences $B_{i}$ and $B_{j}$ s.t. $B_{i}=B_{j}$ but $v\left(B_{i}\right) \neq v\left(B_{j}\right)$, against the hypothesis that $v$ is a CC-evaluation: no $C C$-evaluation assigns two different values to the same sentence, because CCevaluations are functions.

- Assume by the inductive hypothesis (IH) that the (incomplete) tableau $\mathcal{T}_{n}$ whose root is $B_{1}: v\left(B_{1}\right) ; \ldots ; B_{k}: v\left(B_{k}\right)$ and that has height $n$ is open, and that its open branches induce quasi-CC-evaluations. Suppose also (in contradiction with the lemma to be shown) that the tableau $\mathcal{T}_{n+1}$ of height $n+1$ resulting by applying one tableau rule to the terminal nodes of $\mathcal{T}_{n}$ is closed. We reason by 
cases, according to the last rule applied to the nodes in a branch of $\mathcal{T}_{n}$ (we only do two cases):

$(\wedge)$ Suppose a conjunction rule is applied to a node $v$ occurring in an open branch $\mathcal{B}_{n}$ of height $n$ in $\mathcal{T}_{n}$, and all the branches of height $n+1$ resulting from this application are closed. There are three possibilities: $\mathrm{v}$ has in its label $A \wedge B: 1$, or $A \wedge B: 0$, or $A \wedge B: 1 / 2$.

- If $\mathrm{v}$ has $A \wedge B: 1$ in its label, then there is exactly one successor node $\mathrm{v}_{1}$ in the resulting branch $\mathcal{B}_{n+1}$ of height $n$, and $\mathrm{v}_{1}$ has $A: 1 ; B: 1$ in its label. If $\mathcal{B}_{n+1}$ is closed as a result of the addition of $v_{1}$, this means that there is at least one node $\mathrm{w}$, a predecessor of $\mathrm{v}$, such that:

$$
\begin{aligned}
& \text { w has } A: 0 \text { in its label, or } \\
& \text { w has } A: 1 / 2 \text { in its label, or } \\
& \text { w has } B: 0 \text { in its label, or } \\
& \text { w has } B: 1 / 2 \text { in its label }
\end{aligned}
$$

Since we assumed that $\mathcal{B}_{n+1}$ is closed, $\mathcal{B}_{n}$ has a node (namely $\mathrm{v}$ ) that has $A \wedge B: 1$ in its label, and a node (namely w) whose label is as in one of the cases just listed. By $\mathrm{IH}, \mathcal{B}_{n}$ induces a quasi-CC-evaluation. But no quasiCC-evaluation assigns value 1 to a conjunction and a value different from 1 to both conjuncts. Contradiction.

- If v has $A \wedge B: 0$ or $A \wedge B: 1 / 2$ in its label, the reasoning is exactly analogous to the previous case.

$(\rightarrow)$ Suppose a conditional rule is applied to a node $\mathrm{v}$ occurring in an open branch $\mathcal{B}_{n}$ of height $n$ in $\mathcal{T}_{n}$, and all the branches of height $n+1$ resulting from this application are closed. There are three possibilities: $\mathrm{v}$ has in its label $A \rightarrow B: 1$, or $A \rightarrow B: 0$, or $A \rightarrow B: 1 / 2$.

- If $\mathrm{v}$ has $A \rightarrow B: 1 / 2$ in its label, then there are exactly two branches $\mathcal{B}_{n+1}^{1}$ and $\mathcal{B}_{n+1}^{2}$ of height $n+1$ extending $\mathcal{B}_{n}$ with three successor nodes of $\mathrm{v}$, call them $\mathrm{v}_{1}$ and $\mathrm{v}_{2}$, such that:

$$
\begin{aligned}
& \mathrm{v}_{1} \text { has } A: 0 \text { in its label } \\
& \mathrm{v}_{2} \text { has } B: 1 / 2 \text { in its label }
\end{aligned}
$$

Since we assumed that $\mathcal{B}_{n+1}^{1}$ and $\mathcal{B}_{n+1}^{2}$ are both closed, then $\mathcal{B}_{n}$ has two nodes $\mathrm{w}_{1}$ and $\mathrm{w}_{2}$, predecessors of $\mathrm{v}$, such that:

$\mathrm{w}_{1}$ has $A: 1$ or $A: 1 / 2$ in its label

w2 has $B: 0$ or $B: 1$ in its label 
By $\mathrm{IH}, \mathcal{B}_{n}$ induces a quasi-CC-evaluation. But no quasiCC-evaluation assigns value $1 / 2$ to a conditional while assigning any of the following pairs of values to its antecedent and consequent respectively: $\langle 1,0\rangle,\langle 1,1\rangle$, $\langle 1 / 2,0\rangle$, and $\langle 1 / 2,1\rangle$. Contradiction.

- If $\mathrm{v}_{0}$ has $A \rightarrow B: 1$ or $A \rightarrow B: 0$ in its label, the reasoning is exactly analogous to the previous case.

Proposition 2.6 (Soundness) For every finite set $\Gamma$ offormulae and every formula A:

$$
\text { if } \Gamma \vdash_{\mathrm{cc} / \mathrm{TTt}} A \text {, then } \Gamma \models \mathrm{cc} / \mathrm{TT} A
$$

Proof We prove the contrapositive. Suppose that $\Gamma \not \nvdash_{\mathrm{CC} / \mathrm{TT}} A$, for $\Gamma=\left\{B_{1}, \ldots, B_{k}\right\}$. Then there is at least one CC-evaluation $v$ such that $v\left(B_{1}\right) \in\{1,1 / 2\}, \ldots, v\left(B_{k}\right) \in$ $\{1,1 / 2\}$ but $v(A)=0$. Then, by Lemma 2.5 , the tree whose root is labeled as

$$
B_{1}: v\left(B_{1}\right) ; \ldots ; B_{k}: v\left(B_{k}\right) ; A: 0
$$

is open. Therefore, not all the trees whose root is labeled as

$$
B_{1}: i ; \ldots ; B_{k}: j ; A: 0
$$

where $i, j \in\{1,1 / 2\}$, are closed. But this means that $\Gamma \nvdash_{\mathrm{cc} / \mathrm{TTt}} A$.

We finally show that CC/TTt is complete with respect to CC/TT-validity (for inferences with finite sets of premises).

Lemma 2.7 Every open branch of a completed CC/TTt-tableau induces a quasiCC-evaluation that has all the formulae appearing in the branch as its domain and assigns to such formulae the values assigned in the labels appearing in the branch.

Proof (Sketch) Let $\mathcal{T}$ be a completed CC/TTt-tableau with $\mathcal{B}$ an open branch. The branch is finite and it has a unique terminal node $\mathrm{v}$ of the form $p_{i}: k$. Consider now the partial function that only sends $p_{i}$ to $k$ (i.e., that is constituted by the single pair $\left.\left\langle p_{i}, k\right\rangle\right)$. This is clearly a quasi-CC-evaluation. Call this function $v_{0}^{\mathcal{B}}$. Then construct a new function $v_{1}^{\mathcal{B}}$ that simply adds to $v_{0}^{\mathcal{B}}$ every pair $\langle A, k\rangle$, where $A: k$ is in the label of the predecessor of $\mathrm{v}$ in $\mathcal{B}$. More generally, let $v_{n+1}^{\mathcal{B}}$ be the function that results from adding to $v_{n+1}^{\mathcal{B}}$ all the pairs of sentences and values (recall that functions are extensionally construed as pairs) $\langle A, k\rangle$ such that $A: k$ is in the label of the predecessor of $\mathrm{v}$ in $\mathcal{B}$. Proceed in this fashion until the root of $\mathcal{T}$ is reached. It is easy to show that the resulting function $v_{n}^{\mathcal{B}}$ (for $n+1$ the length of $\mathcal{B}$ ) is a quasi-CCevaluation. ${ }^{3}$

\footnotetext{
${ }^{3}$ More precisely, the construction of $v_{n}^{\mathcal{B}}$ is a positive elementary definition that closes at ordinal stage $\omega$. That $v_{n}^{\mathcal{B}}$ is a quasi-CC-evaluation follows by a straightforward induction, similar to the one used in the proof of Lemma 2.5 .
} 
Proposition 2.8 (Completeness) For every finite set $\Gamma$ of formulae and every formula A:

$$
\text { if } \Gamma \models \mathrm{cC} / \mathrm{TT} A \text {, then } \Gamma \vdash_{\mathrm{CC} / \mathrm{TTt}} A
$$

Proof We prove the contrapositive. Assume $\Gamma \nvdash_{\mathrm{cc} / \mathrm{TTt}} A$. By definition this means that not all the CC/TTt-tableaux whose root is labeled as

$$
B_{1}: i ; \ldots ; B_{k}: j ; A: 0
$$

are closed, where $i, j \in\{1,1 / 2\}$. Therefore, at least one such tableau is open. Let $\mathcal{B}$ be an open branch in that tree. By Lemma $2.7, \mathcal{B}$ induces a quasi-CC-evaluation $v^{\mathcal{B}}$ such that

$$
v^{\mathcal{B}}\left(B_{1}\right) \in\{1,1 / 2\}, \ldots, v^{\mathcal{B}}\left(B_{k}\right) \in\{1,1 / 2\}, \text { and } v^{\mathcal{B}}(A)=0 .
$$

Then $v^{\mathcal{B}}$ can be extended to at least one CC-evaluation using Zorn's Lemma. Call one such evaluation $v . v$ and $v^{\mathcal{B}}$ agree on $\Gamma$ and $A$, and therefore

$$
v\left(B_{1}\right) \in\{1,1 / 2\}, \ldots, v\left(B_{k}\right) \in\{1,1 / 2\} \text {, and } v(A)=0 .
$$

But this means that $\Gamma \not \models_{\mathrm{CC} / \mathrm{TT}} A$.

\subsection{Tableau Calculus for DF/TT}

The tableau calculus for DF/TT, in symbols DF/TTt, is given by the rules of CC/TTt, with the conditional rule replaced by the following one:

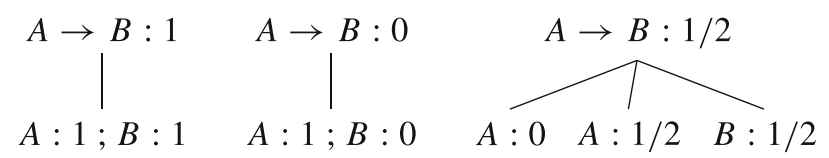

The notions of DF/TTt- $n$-tableau, open and closed branch and open and closed $n$-tableau, and DF/TTt-deducibility (in symbols $\vdash_{\mathrm{DF} / \mathrm{TTt}}$ ) are easily adapted from the corresponding definitions for CC/TTt (Definitions 2.1-2.3).

Proposition 2.9 (Soundness and completeness) For every finite set $\Gamma$ of formulae and every formula $A$ :

\section{$\Gamma \vdash_{\mathrm{DF} / \mathrm{TTt}} A$ if and only if $\Gamma \models_{\mathrm{DF} / \mathrm{TT}} A$}

The proof is entirely similar to the proof of Propositions 2.6 and 2.8.

As mentioned at the beginning of Section 2, tableau calculi are very close to truth table semantics. They are also quite informative: their construction determines all the possible truth value assignments that follow from the hypothesis that a given inference is valid. However, tableau calculi are not a particularly convenient formalism to work with. First, since tableau calculi are refutation calculi, in order to show that $A$ follows from $\Gamma$ in a tableau system, one has to show that the hypothesis that $\Gamma$ holds while $A$ doesn't cannot be maintained. In a classical setting, this amounts to showing that it is not the case that all the sentences in $\Gamma$ can be assigned value 1 while $A$ is assigned value 0 by the corresponding tableau. However, in CC/TT and 
DF/TT we have three values, two of which are designated, so this is not enough: we have to exclude that all the sentences in $\Gamma$ can be assigned a designated value, that is either 1 or $1 / 2$, while $A$ is assigned value 0 . And this requires to consider all the possible combinations of assignments of values 1 and $1 / 2$ to sentences in $\Gamma$ (keeping the assignment of value 0 to $A$ fixed). Of course, as soon as $\Gamma$ contains more than one sentence, showing that $A$ follows from $\Gamma$ requires more than one tableau-more precisely, it requires $2^{k}$ tableaux, for $k$ the cardinality of $\Gamma$.

One might avoid this specific problem by considering the conjunction of all the sentences in $\Gamma$, in symbols $\bigwedge \Gamma$, but this move would not really make the calculus more convenient to work with: the single tableau corresponding to $\bigwedge \Gamma$ would be as complex as the set of tableaux generated by considering all the possible assignments of designated values to sentences in $\Gamma$.

For these reasons, we now present another formalism to capture CC/TT- and DF/TT-validity: many-sided sequent calculi, in particular three-sided sequent calculi. Three-sided sequent calculi are a generalization of standard sequent calculi: instead of building derivation trees labeled with sequents, the rules of the calculus generate derivation trees labeled with triples of sets of sentences, called three-sided sequents. Unlike tableaux, sequent calculi are not refutation calculi, and therefore any derivation of $A$ from $\Gamma$ establishes that $A$ is provable from $\Gamma$. In addition, sequent calculi handle arbitrary sets of premises, including infinite ones. They can also handle (possibly infinite) sets of conclusions, and therefore generalize CC/TT- and DF/TT-validity to multiple conclusions. All these advantages have little costs for the intuitiveness of the calculus. Even though one cannot represent in a sequent calculus all the possible outcomes of assigning a given value to a set of sentences, the sequent rules that we are going to use are very close to the tableau rules, and mirror closely the evaluations of their target sentences according to the CC and DF truth tables.

\section{Three-Sided Sequent Calculi}

In this section, we introduce sound and complete three-sided sequent calculi for CC/TT and DF/TT. Since both CC/TT and DF/TT are super-logics of LP (they extend the latter with a new conditional), we can obtain a sequent calculus by extending an existing calculus for LP, in particular the three-sided sequent axiomatization of LP provided by Ripley [38], itself drawing on techniques introduced by Baaz et al. in $[3] .{ }^{4} \mathrm{~A}$ three-sided sequent, or a sequent for short, is an object of the form

$$
\Gamma|\Delta| \Sigma
$$

where $\Gamma, \Delta$, and $\Sigma$ are sets of formulae. As above, we focus on the calculus for $\mathrm{CC} / \mathrm{TT}$, and then indicate how to adapt it to the case of DF/TT.

\footnotetext{
${ }^{4} \mathrm{LP}$, for the 'Logic of Paradox', is a paraconsistent logic adopted in some approaches to the semantic paradoxes. It is the sub-logic of both CC/TT and DF/TT that results from removing the conditional $(\rightarrow)$ from the latter, and it is therefore quite natural to axiomatize CC/TT and DF/TT over axiomatizations of LP. For more on LP and some of its developments, see Asenjo [2], Priest [33, 34], Goodship [18] and Beall [7].
} 


\subsection{Three-Sided Sequent Calculus for CC/TT}

Let CC/TTm be the calculus given by the following principles:

Axiom:

Rules:

$$
\overline{\Gamma, A|\Delta, A| \Sigma, A} \text { SRef }
$$

$$
\begin{aligned}
& \frac{\Gamma, A|\Delta| \Sigma \quad \Gamma|\Delta, A| \Sigma}{\Gamma|\Delta| \Sigma} \text { cut-a } \frac{\Gamma, A|\Delta| \Sigma \quad \Gamma|\Delta| \Sigma, A}{\Gamma|\Delta| \Sigma} \text { cut-b } \\
& \frac{\Gamma|\Delta, A| \Sigma \quad \Gamma|\Delta| \Sigma, A}{\Gamma|\Delta| \Sigma} \text { cut-c } \\
& \frac{\Gamma|\Delta| \Sigma, A}{\Gamma, \neg A|\Delta| \Sigma} \neg-0 \quad \frac{\Gamma|\Delta, A| \Sigma}{\Gamma|\Delta, \neg A| \Sigma, A} \neg-1 / 2 \quad \frac{\Gamma, A|\Delta| \Sigma}{\Gamma|\Delta| \Sigma, \neg A} \neg-1 \\
& \frac{\Gamma, A, B|\Delta| \Sigma}{\Gamma, A \wedge B|\Delta| \Sigma} \wedge-0 \quad \frac{\Gamma|\Delta, A| \Sigma, A \quad \Gamma|\Delta, B| \Sigma, B \quad \Gamma|\Delta, A, B| \Sigma}{\Gamma|\Delta, A \wedge B| \Sigma} \wedge-1 / 2 \\
& \frac{\Gamma|\Delta| \Sigma, A \quad \Gamma|\Delta| \Sigma, B}{\Gamma|\Delta| \Sigma, A \wedge B} \wedge-1 \\
& \frac{\Gamma|\Delta, A| \Sigma, A \quad \Gamma, B|\Delta| \Sigma}{\Gamma, A \rightarrow B|\Delta| \Sigma} \rightarrow-0 \quad \frac{\Gamma, A|\Delta, B| \Sigma}{\Gamma|\Delta, A \rightarrow B| \Sigma} \rightarrow-1 / 2 \\
& \frac{\Gamma|\Delta, A| \Sigma, A \quad \Gamma|\Delta| \Sigma, B}{\Gamma|\Delta| \Sigma, A \rightarrow B} \rightarrow-1
\end{aligned}
$$

Three-sided sequents have an immediate semantic reading. Consider a sequent $\Gamma|\Delta| \Sigma$. Intuitively, sentences in $\Gamma$ should be thought of as having value 0 , sentences in $\Delta$ should be thought of as having value $1 / 2$, and sentences in $\Sigma$ should be thought of as having value 1 . This makes it easy to understand the rationale behind the sequent rules. For example, consider the rule $\neg-0$ : if $A$ is thought of as having value 1 (i.e., it appears in the rightmost position in a sequent), then $\neg A$ is thought of as having value 0 (and is therefore placed in the leftmost position in the sequent). Similar considerations apply to all the other sequent rules.

A derivation of a sequent $\Gamma|\Delta| \Sigma$ in CC/TTm is a tree labeled with sequents, whose leaves are axioms of CC/TTm and whose remaining nodes are obtained from their predecessors by applying the CC/TTm-rules. Let $\Gamma \vdash_{\mathrm{CC} / \mathrm{TTm}} \Delta$ be a shorthand for 'there is a derivation of $\Gamma|\Delta| \Delta$ in CC/TTm'.

Definition 3.1 (Satisfaction and Validity) A C-evaluation $v$ satisfies a sequent $\Gamma|\Delta| \Sigma$ if:

- there is an $A \in \Gamma$ s.t. $v(A)=0$, or

- there is a $B \in \Delta$ s.t. $v(B)=1 / 2$, or

- there is a $C \in \Sigma$ s.t. $v(C)=1$.

A C-evaluation $v$ is a countermodel for a sequent $\Gamma|\Delta| \Sigma$ if $v$ does not satisfy it. A sequent $\Gamma|\Delta| \Sigma$ is CC/TT-valid if it is satisfied by every CC/TT-evaluation. 
Let's expand the notion of CC/TT-validity to allow for multiple conclusion, and say that $\Gamma \models \mathrm{CC} / \mathrm{TT} \Delta$ if every $\mathrm{CC}$-evaluation that makes all sentences of $\Gamma \mathrm{T}$-true makes at least one sentence in $\Delta \mathrm{T}$-true. The following lemma, adapted from [38], is immediate from the definition of satisfaction and validity.

Lemma 3.2 For all sets of formulae $\Gamma$ and $\Delta$ :

\section{$\Gamma \models \mathrm{CC} / \mathrm{TT} \Delta$ if and only if $\Gamma|\Delta| \Delta$ is $\mathrm{CC} / \mathrm{TT}$-valid}

Before establishing soundness and completeness for CC/TTm, we provide an example of how one can reason with this calculus. More precisely, we show the equivalence of $A \rightarrow \neg B$ and $\neg(A \rightarrow B)$ within it. By the above lemma, this amounts to deriving the sequents $\neg(A \rightarrow B)|A \rightarrow \neg B| A \rightarrow \neg B$ and $A \rightarrow \neg B|\neg(A \rightarrow B)|$ $\neg(A \rightarrow B)$. In the following examples, we use the empty set symbol $\varnothing$ only in order to make the derivations more readable. The following derivation establishes the first sequent:

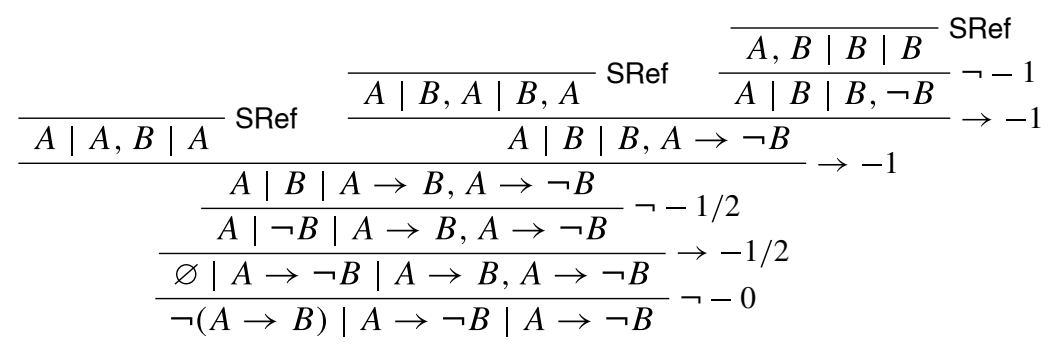

The following derivation establishes the second sequent:

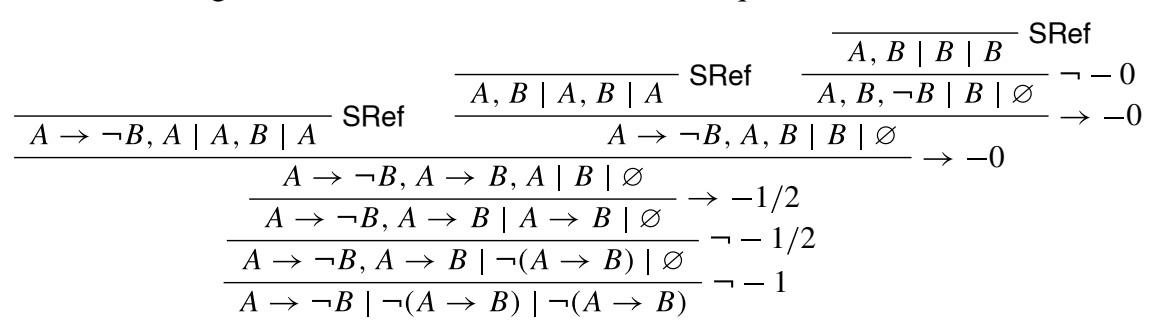

We now proceed to establish soundness and completeness for CC/TTm.

Proposition 3.3 (Soundness) If $\Gamma \vdash_{\mathrm{cc} / \mathrm{TTm}} \Delta$, then $\Gamma \models_{\mathrm{DF} / \mathrm{TT}} \Delta$.

Proof By induction on the length of the derivation of $\Gamma|\Delta| \Delta$.

To prove completeness, we prove the following more general result.

Proposition 3.4 For every triple of sets of formulae $\Gamma, \Delta$, and $\Sigma$, exactly one of the two following cases is given:

- there is a derivation of $\Gamma|\Delta| \Sigma$ in CC/TTm

- $\quad \Gamma|\Delta| \Sigma$ has a countermodel. 
Proof We employ the method of Schütte's search trees, adapted to CC/TTm. ${ }^{5}$ For every sequent $\Gamma|\Delta| \Sigma$, such method provides the means to construct a tree labeled with sequents which either constitutes a derivation of $\Gamma|\Delta| \Sigma$ in CC/TTm or can be used to extract a countermodel to $\Gamma|\Delta| \Sigma$.

We begin by defining three inductive jumps, that extend a given directed tree labeled with sequents by applying all the rules of CC/TTm. Formally, such a tree is constituted by a pair $\langle\mathrm{N}, \mathrm{S}\rangle$, where $\mathrm{N}$ is the set of nodes and $\mathrm{S}$ is the set of edges, together with a labeling function, that is, a function from $\mathrm{N}$ to their labels (that is, sequents). To simplify our presentation, we identify nodes with their labels, and pairs of nodes with pairs of labels. For every labeled directed tree $\langle\mathrm{N}, \mathrm{S}\rangle$, define the following sets by positive elementary induction:

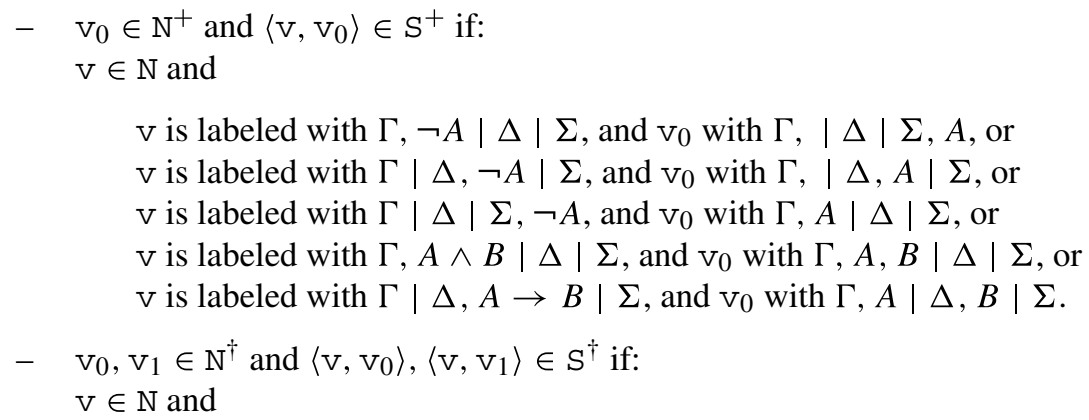

$\mathrm{v}$ is labeled with $\Gamma|\Delta| \Sigma, A \wedge B, \mathrm{v}_{0}$ is labeled with $\Gamma|\Delta| \Sigma, A$, and $\mathrm{v}_{1}$ with $\Gamma|\Delta| \Sigma, B$, or

$\mathrm{v}$ is labeled with $\Gamma|\Delta| \Sigma, A \rightarrow B, \mathrm{v}_{0}$ is labeled with $\Gamma|\Delta, A| \Sigma, A$, and $\mathrm{v}_{1}$ with $\Gamma|\Delta| \Sigma, B$, or

$\mathrm{v}$ is labeled with $\Gamma, A \rightarrow B|\Delta| \Sigma, \mathrm{v}_{0}$ is labeled with $\Gamma|\Delta, A| \Sigma, A$, and $\mathrm{v}_{1}$ with $\Gamma, B|\Delta| \Sigma$.

- $\mathrm{v}_{0}, \mathrm{v}_{1}, \mathrm{v}_{2} \in \mathrm{N}^{\ddagger}$ and $\left\langle\mathrm{v}, \mathrm{v}_{0}\right\rangle,\left\langle\mathrm{v}, \mathrm{v}_{1}\right\rangle,\left\langle\mathrm{v}, \mathrm{v}_{2}\right\rangle \in \mathrm{S}^{\ddagger}$ if:

$\mathrm{v} \in \mathrm{N}$ and $\mathrm{v}$ is labeled with $\Gamma|\Delta, A \wedge B| \Sigma, \mathrm{v}_{0}$ is labeled with $\Gamma|\Delta, A| \Sigma, A$,

$\mathrm{v}_{1}$ is labeled with $\Gamma|\Delta, B| \Sigma, B$, and $\mathrm{v}_{2}$ with $\Gamma|\Delta, A, B| \Sigma$, or

$\mathrm{v} \in \mathrm{N}$ and $\mathrm{v}$ is labeled with $\Gamma|\Delta| \Sigma, \mathrm{v}_{0}$ is labeled with $\Gamma, A \mid \Delta$,

$A \mid \Sigma, \mathrm{v}_{1}$ is labeled with $\Gamma, A|\Delta| \Sigma, A$, and $\mathrm{v}_{2}$ with $\Gamma|\Delta, A| \Sigma, A$.

Informally, one can see the jumps ${ }^{+},{ }^{\dagger}$, and ${ }^{\dagger}$ as corresponding to the operations of extending a given labeled tree to another labeled tree, where the sequents that are added result from applying the rules of CC/TTm 'upside down', that is, going from a sequent to all its possible premises according to the CC/TTm rules. ${ }^{6}$

\footnotetext{
${ }^{5}$ Search trees were originally introduced in Schutte [39]. See Pohlers [32, Chapter 4] for an application of this method to one-sided sequents for classical logic, Baaz, Fermüller, and Zach [3, 4] for generalizations and applications to $n$-sided sequents, and Ripley [38] for an application to ST.

${ }^{6}$ The cut-rules are applied 'simultaneously', as in a single combined rule. See Ripley [38], p. 366.
} 
Now we construct a search tree for every sequent, that is, a labeled tree where the above jumps are systematically applied as many times as possible. For every sequent $\Gamma|\Delta| \Sigma$ define (for a limit ordinal $\delta$ ):

$$
\begin{aligned}
\mathrm{N}_{0} & :=\Gamma|\Delta| \Sigma & \mathrm{S}_{0} & :=\varnothing \\
\mathrm{N}_{\alpha+1} & :=\left(\mathrm{N}_{\alpha}\right)^{+} \cup\left(\mathrm{N}_{\alpha}\right)^{\dagger} \cup\left(\mathrm{N}_{\alpha}\right)^{\ddagger} & \mathrm{S}_{\alpha+1} & :=\left(\mathrm{S}_{\alpha}\right)^{+} \cup\left(\mathrm{S}_{\alpha}\right)^{\dagger} \cup\left(\mathrm{S}_{\alpha}\right)^{\ddagger} \\
\mathrm{N}_{\delta} & :=\bigcup_{\alpha<\delta} \mathrm{N}_{\alpha} & \mathrm{S}_{\delta} & :=\bigcup_{\alpha<\delta} \mathrm{S}_{\alpha}
\end{aligned}
$$

Finally, define (where Ord is the class of all ordinals): ${ }^{7}$

$$
\mathrm{N}_{\infty}:=\bigcup_{\alpha \in \text { Ord }} \mathrm{N}_{\alpha} \mathrm{S}_{\infty}:=\bigcup_{\alpha \in \text { Ord }} \mathrm{S}_{\alpha}
$$

The tree $\left\langle\mathrm{N}_{\infty}, \mathrm{S}_{\infty}\right\rangle$ is the search tree for $\Gamma|\Delta| \Sigma$. We say that $\left\langle\mathrm{N}_{\infty}, \mathrm{S}_{\infty}\right\rangle$ is closed if all its branches have finite length and have an axiom of CC/TTm as their topmost nodes, and that it is open otherwise. Clearly, if $\left\langle\mathrm{N}_{\infty}, \mathrm{S}_{\infty}\right\rangle$ is closed, this very tree provides a proof of $\Gamma|\Delta| \Sigma$, since its topmost nodes are axioms and all the other nodes are obtained from their predecessors by applying CC/TTm-rules. Now we show that if $\left\langle\mathrm{N}_{\infty}, \mathrm{S}_{\infty}\right\rangle$ is open, one can use it to construct a countermodel for $\Gamma|\Delta| \Sigma$.

Suppose $\left\langle\mathrm{N}_{\infty}, \mathrm{S}_{\infty}\right\rangle$ is open, and let $\mathcal{B}$ be an open branch in it. Let $\Gamma_{\infty}\left|\Delta_{\infty}\right| \Sigma_{\infty}$ be the sequent defined as the union of all the sequents in $\mathcal{B}$. More formally:

$$
\Gamma_{\infty}:=\bigcup_{\Gamma_{0}\left|\Delta_{0}\right| \Sigma_{0} \in \mathcal{B}} \Gamma_{0} \Delta_{\infty}:=\bigcup_{\Gamma_{1}\left|\Delta_{1}\right| \Sigma_{1} \in \mathcal{B}} \Delta_{1} \Sigma_{\infty}:=\bigcup_{\Gamma_{2}\left|\Delta_{2}\right| \Sigma_{2} \in \mathcal{B}} \Sigma_{2}
$$

We now have to show that no formula is in $\Gamma_{\infty} \cap \Delta_{\infty} \cap \Sigma_{\infty}$ (otherwise the branch we are constructing might not provide a countermodel). Suppose that there is a formula $A$ and there are sequents $\Gamma_{0}\left|\Delta_{0}\right| \Sigma_{0}, \Gamma_{1}\left|\Delta_{1}\right| \Sigma_{1}$, and $\Gamma_{2}\left|\Delta_{2}\right| \Sigma_{2}$ such that $A \in \Gamma_{0} \cap \Delta_{1} \cap \Sigma_{2}$. We reason by cases, in order to reach contradictions:

- Suppose $A$ is a propositional variable $p_{i}$. Since $\Gamma_{0}\left|\Delta_{0}\right| \Sigma_{0}, \Gamma_{1}\left|\Delta_{1}\right| \Sigma_{1}$, and $\Gamma_{2}\left|\Delta_{2}\right| \Sigma_{2}$ all belong to the same open branch $\mathcal{B}$, then they occur at different heights within $\mathcal{B}$. Suppose without loss of generality that $\Gamma_{0}\left|\Delta_{0}\right| \Sigma_{0}$ occurs at height $n$ (counting upwards the nodes appearing in $\mathcal{B}$ starting from the lowest node, labeled with $\Gamma|\Delta| \Sigma$ ), that $\Gamma_{1}\left|\Delta_{1}\right| \Sigma_{1}$ occurs at height $n+j$, and that $\Gamma_{2}\left|\Delta_{2}\right| \Sigma_{2}$ occurs at height $n+j+k$ (considering different orders would not make a difference). Since $p_{i} \in \Gamma_{0}$ and all the rules of CC/TTm are contextsharing, ${ }^{8} p_{i}$ is 'carried upwards' during the construction of successive stages of $\mathcal{B}$. Therefore, at height $n+j$ we have that $p_{i} \in \Gamma_{1}$ and $p_{i} \in \Delta_{1}$, and at height $n+j+k$ we have that $p_{i} \in \Gamma_{2}, p_{i} \in \Delta_{2}$, and $p_{i} \in \Sigma_{2}$. But this means that $\Gamma_{2}\left|\Delta_{2}\right| \Sigma_{2}$ is an axiom of CC/TTm, and that $\mathcal{B}$ is closed. Contradiction.

\footnotetext{
${ }^{7}$ Since we construct one search tree per sequent, a more perspicuous notation would indicate the dependence of $\mathrm{N}_{\infty}$ on the starting sequent $\Gamma|\Delta| \Sigma$, for example by writing $\mathrm{N}_{\infty}(\Gamma|\Delta| \Sigma)$ (similarly for $\mathrm{S}_{\infty}$ and the ordinal stages). We stick to the simpler notation for readability and because the sequent in question is clear from the context.

${ }^{8}$ That is, sequent rules with more than one premises are applied to sequents with identical side-formulae, i.e., sharing the sets of premises $\Gamma, \Delta$, and $\Sigma$. See Troelstra and Schwichtenberg [41, 64 and following] for more details.
} 
- Suppose $A$ is a complex formula of complexity $n+1$, and assume the claim as IH for formulae of complexity up to $n .{ }^{9}$ Suppose $A$ is $B \rightarrow C$, and that $\Gamma_{0}\left|\Delta_{0}\right| \Sigma_{0}$ occurs at height $n$, that $\Gamma_{1}\left|\Delta_{1}\right| \Sigma_{1}$ occurs at height $n+j$, and that $\Gamma_{2}\left|\Delta_{2}\right| \Sigma_{2}$ occurs at height $n+j+k$. Then:

* $\quad B \in \Delta_{0}^{\prime} \cap \Sigma_{0}^{\prime}$ and $C \in \Gamma_{0}^{\prime \prime}$ where $\Gamma_{0}^{\prime}\left|\Delta_{1}^{\prime}\right| \Sigma_{0}^{\prime}$ and $\Gamma_{0}^{\prime \prime}\left|\Delta_{0}^{\prime \prime}\right| \Sigma_{0}^{\prime \prime}$ are predecessors of $\Gamma_{0}\left|\Delta_{0}\right| \Sigma_{0}$ in $\left\langle\mathrm{N}_{\infty}, \mathrm{S}_{\infty}\right\rangle$, and one of them is in $\mathcal{B}$.

* $\quad B \in \Gamma_{1}^{\prime}$ and $C \in \Delta_{1}^{\prime}$ where $\Gamma_{1}^{\prime}\left|\Delta_{1}^{\prime}\right| \Sigma_{1}^{\prime}$ is a predecessor of $\Gamma_{1}\left|\Delta_{1}\right| \Sigma_{1}$ in $\left\langle\mathrm{N}_{\infty}, \mathrm{S}_{\infty}\right\rangle$, and is in $\mathcal{B}$.

* $\quad B \in \Delta_{2}^{\prime} \cap \Sigma_{2}^{\prime}$ and $C \in \Sigma_{2}^{\prime \prime}$ where $\Gamma_{2}^{\prime}\left|\Delta_{2}^{\prime}\right| \Sigma_{2}^{\prime}$ and $\Gamma_{2}^{\prime \prime}\left|\Delta_{2}^{\prime \prime}\right| \Sigma_{2}^{\prime \prime}$ are predecessors of $\Gamma_{2}\left|\Delta_{2}\right| \Sigma_{2}$ in $\left\langle\mathrm{N}_{\infty}, \mathrm{S}_{\infty}\right\rangle$, and one of them is in $\mathcal{B}$.

Therefore, one of the following is the case:

(i) $B \in \Gamma_{1}^{\prime} \cap \Delta_{0}^{\prime} \cap \Sigma_{0}^{\prime}$, where $\Gamma_{1}^{\prime} \subseteq \Gamma_{\infty}, \Delta_{0}^{\prime} \subseteq \Delta_{\infty}$, and $\Sigma_{0}^{\prime} \subseteq \Sigma_{\infty}$; or

(ii) $B \in \Gamma_{1}^{\prime} \cap \Delta_{2}^{\prime} \cap \Sigma_{2}^{\prime}$, where $\Gamma_{1}^{\prime} \subseteq \Gamma_{\infty}, \Delta_{2}^{\prime} \subseteq \Delta_{\infty}$, and $\Sigma_{2}^{\prime} \subseteq \Sigma_{\infty}$; or

(iii) $C \in \Gamma_{0}^{\prime \prime} \cap \Delta_{1}^{\prime} \cap \Sigma_{2}^{\prime \prime}$, where $\Gamma_{0}^{\prime \prime} \subseteq \Gamma_{\infty}, \Delta_{1}^{\prime} \subseteq \Delta_{\infty}$, and $\Sigma_{2}^{\prime \prime} \subseteq \Sigma_{\infty}$;

But all of (i)-(iii) contradict our IH. The cases of the other connectives are similar.

Now that we have shown that the open branch $\mathcal{B}$ is such that the union sets of the left-, middle-, and right-items in the sequents in $\mathcal{B}$ have an empty intersection, that is that no formula is in $\Gamma_{\infty} \cap \Delta_{\infty} \cap \Sigma_{\infty}$, we can proceed to extract a proper countermodel from $\Gamma_{\infty}, \Delta_{\infty}$, and $\Sigma_{\infty}$. In order to construct our countermodel, we now define the following partial function from formulae to $\{0,1 / 2,1\}$ by simultaneous induction:

$$
\begin{aligned}
& w_{\mathcal{B}}^{0}\left(p_{i}\right):=\left\{\begin{array}{l}
0, \text { if } p_{i} \notin \Gamma_{\infty} \text { and } p \in \Sigma_{\infty} \\
1 / 2, \text { if } p_{i} \notin \Delta_{\infty} \text { and } p \in \Gamma_{\infty} \\
1, \text { if } p_{i} \notin \Sigma_{\infty} \text { and } p \in \Delta_{\infty}
\end{array}\right. \\
& 0 \text {, if }\left\{\begin{array}{c}
A \text { is } \neg B \text { and } w_{\mathcal{B}}^{\alpha}(B)=1, \text { or } \\
A \text { is } B \wedge C \text { and } w_{\mathcal{B}}^{\alpha}(B)=0 \text { or } w_{\mathcal{B}}^{\alpha}(C)=0, \text { or } \\
A \text { is } B \rightarrow C \text { and } w_{\mathcal{B}}^{\alpha}(B)=1 \text { or } w_{\mathcal{B}}^{\alpha}(B)=1 / 2, \\
\text { and } w_{\mathcal{B}}^{\alpha}(C)=0
\end{array}\right.
\end{aligned}
$$

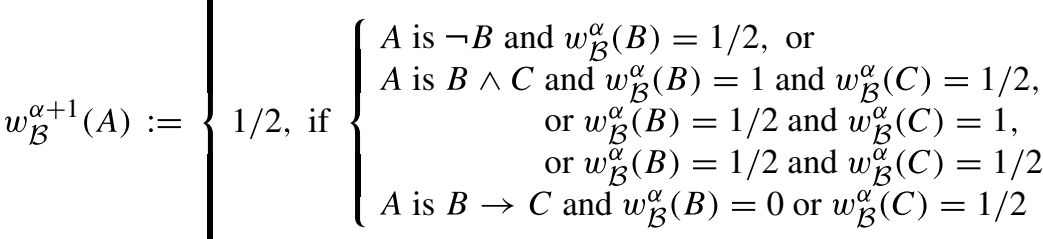

$$
\begin{aligned}
& 1 \text {, if }\left\{\begin{array}{c}
A \text { is } \neg B \text { and } w_{\mathcal{B}}^{\alpha}(B)=0 \text {, or } \\
A \text { is } B \wedge C \text { and } w_{\mathcal{B}}^{\alpha}(B)=1 \text { and } w_{\mathcal{B}}^{\alpha}(C)=1 \text {, or } \\
A \text { is } B \rightarrow C \text { and } w_{\mathcal{B}}^{\alpha}(B)=1 \text { or } w_{\mathcal{B}}^{\alpha}(B)=1 / 2, \\
\text { and } w_{\mathcal{B}}^{\alpha}(C)=1
\end{array}\right.
\end{aligned}
$$

\footnotetext{
${ }^{9}$ Since the cut-rules only move formulae upwards, their case is covered by the case of logical connectives done here in detail. See Ripley [38], pp. 374 and following for more details.
} 
By construction, $w_{\mathcal{B}}$ is a quasi-C-evaluation, ${ }^{10}$ and it can be extended to a $\mathrm{C}$ evaluation $w$ that agrees with $w_{\mathcal{B}}$ on $\Gamma_{\infty} \cup \Delta_{\infty} \cup \Sigma_{\infty}$. Therefore, for every $A \in \Gamma_{\infty}$, $B \in \Delta_{\infty}$, and $C \in \Sigma_{\infty}$ :

$$
w(A) \neq 0 \quad w(B) \neq 1 / 2 \quad w(C) \neq 1
$$

But since $\Gamma \subseteq \Gamma_{\infty}, \Delta \subseteq \Delta_{\infty}$, and $\Sigma \subseteq \Sigma_{\infty}$, for every $A \in \Gamma, B \in \Delta$, and $C \in \Sigma$ :

$$
w(A) \neq 0 \quad w(B) \neq 1 / 2 \quad w(C) \neq 1
$$

Therefore, there is a C-evaluation that does not satisfy $\Gamma|\Delta| \Sigma$, namely $w$.

A few observations on the functions $w_{\mathcal{B}}^{\alpha}$ are in order. First of all, the definition of $w_{\mathcal{B}}^{0}$ is in part arbitrary, as other choices of truth value assignments to propositional variables would have been possible. In order to get a countermodel, one just needs a function that (i) assigns to the propositional variables in $\Gamma_{\infty}, \Delta_{\infty}$, and $\Sigma_{\infty}$ a value that is incompatible with the corresponding position of such variables in the union sequent (and clearly there is more than one choice here) and that (ii) is a quasi-Cevaluation. Notice moreover that the construction of every $w_{\mathcal{B}}^{\alpha}$ is by simultaneous induction, but every $w_{\mathcal{B}}^{\alpha}$ is inductive in $\Gamma_{\infty}, \Delta_{\infty}$, and $\Sigma_{\infty}$, since these sets occur also negatively in the definition of $w_{\mathcal{B}}^{0}{ }^{11}$ This seems unavoidable: there seems to be no definition of 'having value 1 if not in $\Gamma_{\infty}, 1 / 2$ if not in $\Delta_{\infty}$, and 0 if not in $\Sigma_{\infty}$ ' that yields a function and that is positive in $\Gamma_{\infty}, \Delta_{\infty}$, and $\Sigma_{\infty}$. However, this causes no problem as far as the existence and uniqueness of $w_{\mathcal{B}}$ is concerned, since the existence and uniqueness of $\Gamma_{\infty}, \Delta_{\infty}$, and $\Sigma_{\infty}$ is immediate by their definition.

Finally, notice that we gave a simplified inductive construction for $w_{\mathcal{B}}$. More specifically, we define $w_{\mathcal{B}}$ directly as a function rather than as a positive elementary set of pairs of sentences and values (then one would have had to show that such set is, indeed, a function). Giving a proper positive elementary definition of $w_{\mathcal{B}}$ would make it clearer that its construction is by simultaneous induction, but would be significantly less readable.

A completeness theorem for CC/TTm is now immediate from Proposition 3.4.

Proposition 3.5 (Completeness) For every set $\Gamma$ of formulae and every formula A:

$$
\text { if } \Gamma \models \mathrm{cC} / \mathrm{TT} \Delta \text {, then } \Gamma \vdash_{\mathrm{CC} / \mathrm{TTm}} \Delta \text {. }
$$

\subsection{Three-Sided Sequent Calculus for DF/TT}

The three-sided sequent calculus for DF/TT, in symbols DF/TTm, is given by the rules of CC/TTm, with the conditional rules replaced by the following ones:

$$
\frac{\Gamma|\Delta| \Sigma, A \quad \Gamma, B|\Delta| \Sigma}{\Gamma, A \rightarrow B|\Delta| \Sigma} \rightarrow-0 \quad \frac{\Gamma, A|\Delta, A, B| \Sigma}{\Gamma|\Delta, A \rightarrow B| \Sigma} \rightarrow-1 / 2
$$

\footnotetext{
${ }^{10}$ See Definition 2.4. That $w_{\mathcal{B}}$ is a quasi-C-evaluation can be rigorously shown by an induction similar to the one employed in the proof of Lemma 2.5.

${ }^{11}$ See Moschovakis [31, 17 and following].
} 


$$
\frac{\Gamma|\Delta| \Sigma, A \quad \Gamma|\Delta| \Sigma, B}{\Gamma|\Delta| \Sigma, A \rightarrow B} \rightarrow-1
$$

The notions of DF/TTm-derivability, as well as of satisfaction and validity of a three-sided sequent are immediate from the corresponding definitions for CC/TTm (Definition 3.1).

Proposition 3.6 (Soundness and completeness) For every set $\Gamma$ of formulae and every formula $A$ :

$$
\Gamma \vdash_{\mathrm{DF} / \mathrm{TTm}} A \text { if and only if } \Gamma \models \mathrm{DF} / \mathrm{TT} A
$$

The proof is entirely similar to the proof of soundness and completeness for CC/TTm.

\section{Algebraic Semantics}

In this section, we explore the algebraic structures that correspond to DF/TT and $\mathrm{CC} / \mathrm{TT}$, and investigate the prospects for an algebraic semantics of these two logics. We begin by recalling some structures, and introducing the algebraic counterparts of DF/TT. We start with DF/TT because, as will be clear in Section 4.3, algebraically it is significantly more tractable than $\mathrm{CC} / \mathrm{TT}$. We use overlined uppercase Latin letters $(\bar{A}, \bar{B}, \bar{C}, \ldots)$ to range over sets (supports of algebraic structures) in order to avoid possible confusions with meta-variables for $\mathcal{L}_{\rightarrow}$-formulae, and boldface characters to indicate designated elements of the supports of algebraic structures $(\mathbf{1}, \mathbf{0}, \mathbf{1} / \mathbf{2}, \ldots)$, in order to avoid possible confusions with truth values in truth table semantics.

\subsection{De Finetti Algebrae}

We begin by some basic definitions, which will be needed for the algebraic semantics for DF/TT.

Definition 4.1 A structure $\mathcal{A}=\langle\bar{A}, \sqcap, \sqcup, \mathbf{0}, \mathbf{1}\rangle$, where $\bar{A}$ is a set and $\mathbf{0}, \mathbf{1} \in \bar{A}$, is a distributive bounded lattice if for every $a, b, c \in \bar{A}$ :

- The lattice conditions are satisfied:

$$
\begin{aligned}
& a \sqcap b=b \sqcap a \text { and } a \sqcup b=b \sqcup a \text { (commutativity) } \\
& a \sqcap(b \sqcap c)=(a \sqcap b) \sqcap c \text { and } a \sqcup(b \sqcup c)=(a \sqcup b) \sqcup c \text { (associativity) } \\
& a \sqcup(b \sqcap a)=a \text { and } a \sqcap(b \sqcup a)=a \text { (absorption) }
\end{aligned}
$$

- The lattice is bounded:

$$
\begin{aligned}
& a \sqcup \mathbf{0}=a \\
& a \sqcap \mathbf{1}=a
\end{aligned}
$$

- The lattice is distributive:

$$
\begin{aligned}
& a \sqcap(b \sqcup c)=(a \sqcap b) \sqcup(a \sqcap c) \\
& a \sqcup(b \sqcap c)=(a \sqcup b) \sqcap(a \sqcup c)
\end{aligned}
$$


For every lattice $\mathcal{A}=\langle\bar{A}, \sqcap, \sqcup\rangle$ the order induced by $\mathcal{A}$ is the binary relation $\sqsubseteq \subseteq(\bar{A} \times \bar{A})$ such that:

$$
a \sqsubseteq b \text { if and only if } a \sqcap b=a \text { if and only if } a \sqcup b=b .^{12}
$$

An involution on a lattice $\mathcal{A}$ is a unary operation - s.t. for every $a, b \in \bar{A}$ :

$$
\begin{aligned}
& \text { If } a \sqsubseteq b, \text { then }-b \sqsubseteq-a \text {, and } \\
& --a=a
\end{aligned}
$$

A bounded, distributive, involutive lattice $\mathcal{A}=\langle\bar{A}, \sqcap, \sqcup,-, \mathbf{0}, \mathbf{1}\rangle$ is a De Morgan algebra if for every $a, b \in \bar{A}$ :

$$
\begin{aligned}
& -(a \sqcap b)=(-a \sqcup-b), \text { and } \\
& -(a \sqcup b)=(-a \sqcap-b)
\end{aligned}
$$

A De Morgan algebra $\mathcal{A}=\langle\bar{A}, \sqcap, \sqcup,-, \mathbf{0}, \mathbf{1}\rangle$ is Kleene if, for every $a, b \in \bar{A}$ :

$$
a \sqcap-a \sqsubseteq b \sqcup-b
$$

A relative pseudocomplementation on a lattice $\mathcal{A}$ is a binary operation $\longmapsto$ s.t. for every $a, b \in \bar{A}$ :

$a \sqcap c \sqsubseteq b$ if and only if $c \sqsubseteq a \longmapsto b$ for all $c \in \bar{A}$.

A relatively pseudocompletemented Kleene algebra $\mathcal{A}=\langle\bar{A}, \sqcap, \sqcup,-, \longmapsto, \mathbf{0}, \mathbf{1}\rangle$ is an Ł3 algebra if for every $a \in \bar{A}$ :

$$
(a \longmapsto \mathbf{0}) \sqcup(-a \longmapsto a)=\mathbf{1}
$$

An $Ł 3$ algebra $\mathcal{A}=\langle\bar{A}, \sqcap, \sqcup,-, \longmapsto, \longrightarrow, \mathbf{0}, \mathbf{1}, \mathbf{1} / \mathbf{2}\rangle$ is de Finetti if:

There is a distinguished element $\mathbf{1} / \mathbf{2} \in \bar{A}$ s.t. $-\mathbf{1} / \mathbf{2}=\mathbf{1} / \mathbf{2}$, and

There is an operation $\square$ defined on $\bar{A} \times \bar{A}$ s.t. $a-b=(\mathbf{1} / \mathbf{2} \sqcap-a) \sqcup(a \sqcap b) .^{13}$

Some remarks on de Finetti algebrae are in order. First, we have defined them over Ł3-algebrae (also known as Łukasiewicz (or Moisil-Łukasiewicz) trivalent algebrae), but other options are possible, including $\mathrm{MV}_{3}$-algebrae. ${ }^{14}$ We have adopted $€ 3$-algebrae both because they are simpler than $\mathrm{MV}_{n}$-algebrae, and in order to better relate our presentation and results to the elegant formalization and the results of [30]. Second, de Finetti algebrae have a paraconsistent flavour, suggested by the behaviour

\footnotetext{
${ }^{12}$ Notice that $\sqsubseteq$ is transitive. Suppose that $a \sqsubseteq b$ and $b \sqsubseteq c$. Then, $a \sqcup c=(a \sqcap b) \sqcup c=c \sqcup(a \sqcap b)=$ $(c \sqcup a) \sqcap(c \sqcup b)=(c \sqcup a) \sqcap(b \sqcup c)=(c \sqcup a) \sqcap c=c$ (these identities follow from the assumption that $a \sqsubseteq b$, commutativity, distributivity, the assumption that $b \sqsubseteq c$, distributivity again, and absorption, in this order). The proof for $a \sqcap c=a$ is similar. Therefore, $a \sqsubseteq c$.

${ }^{13}$ The definition of $Ł 3$ algebrae follows Milne [30, 517-518], and so does the characterization of the algebraic counterpart of the de Finetti conditional over them. We note that Milne considers algebrae of conditional events, while we consider arbitrary supports. Nothing crucial hinges on this.

${ }^{14}$ See Gottwald [19, Ch. 9.2] and Malinowski [27, Ch. 5]. Indeed, while $\mathrm{MV}_{n}$ algebrae provide suitable algebraic counterparts for every $n$-valued Łukasiewicz logic (and MV-algebrae algebraically characterize Łukasiewicz continuum-valued logic), Łukasiewicz algebrae only succeed in capturing the three- and four-valued cases.
} 
of the element $\mathbf{1 / 2}$. Such flavour is more vividly expressed by noticing that they are both special cases of $L P$ algebrae. In the characterization offered by Pynko [35], a Kleene algebra $\mathcal{A}=\langle\bar{A}, \sqcap, \sqcup,-, \mathbf{0}, \mathbf{1}\rangle$ is $L P$ if it has an inconsistent proper filter on its support, that is, if there is an $\bar{F} \subset \bar{A}$ s.t. for every $a, b, \in \bar{A}$ and for some $c \in \bar{F}$

(i) if $a \in \bar{F}$ and $a \sqsubseteq b$, then $b \in \bar{F}$,

(ii) if $a, b \in \bar{F}$, then $a \sqcap b \in \bar{F}$,

(iii) both $c \in \bar{F}$ and $-c \in \bar{F}$.

It is easily seen that de Finetti algebrae are LP. Let $\mathcal{A}$ be a de Finetti algebra with support $\bar{A}$. The set $\{a \in \bar{A} \mid \mathbf{1} / \mathbf{2} \sqsubseteq a\} \subset \bar{A}$ provides the required inconsistent proper filter.

(i) is immediate, because $\sqsubseteq$ is transitive.

As for (ii), assume that $\mathbf{1} / \mathbf{2} \sqsubseteq a$ and that $\mathbf{1} / \mathbf{2} \sqsubseteq b$. By Definition 4.1 , this assumption entails that $(\mathbf{1} / \mathbf{2} \sqcup a)=a$ and that $(\mathbf{1} / \mathbf{2} \sqcup b)=b$. But since we have $1 / 2 \sqcup(a \sqcap b)=(1 / 2 \sqcup a) \sqcap(1 / 2 \sqcup b)$ by distributivity, we also have $(\mathbf{1} / \mathbf{2} \sqcup a) \sqcap(\mathbf{1} / \mathbf{2} \sqcup b)=a \sqcap b$ by our assumption, that is, $\mathbf{1} / \mathbf{2} \sqsubseteq a \sqcap b$.

As for (iii), notice that both $\mathbf{1} / \mathbf{2}$ and $-\mathbf{1} / \mathbf{2}$ are in $\{a \in \bar{A} \mid \mathbf{1} / \mathbf{2} \sqsubseteq a\}$.

\subsection{Algebraic Semantics for DF/TT}

In order to prove algebraic soundness and completeness for DF/TT, we construct the Lindenbaum-Tarski algebra of a set of formulae, for DF/TTm-deducibility. Therefore, we first isolate the relation of DF/TTm-provable equivalence (where 'equivalence' is formalized via the DF-biconditional).

Definition 4.2 For every $\Gamma \subseteq$ For, let $\sim_{\Gamma}^{\text {df }} \subseteq$ For $\times$ For be the relation defined as follows:

$$
A \sim_{\Gamma}^{\mathrm{df}} B \text { if and only if } \Gamma \vdash_{\mathrm{DF} / \mathrm{TTm}} A \leftrightarrow B
$$

where $\leftrightarrow$ denotes the de Finetti biconditional.

This definition, however, does not partition the set of formulae into equivalence classes, but only into sets that have weaker closure conditions.

Lemma 4.3 In general, $\sim_{\Gamma}^{d f}$ is not an equivalence relation on For $\times$ For.

Proof Reflexivity and symmetry hold, since $\Gamma \vdash_{\mathrm{DF} / \mathrm{TTm}} A \leftrightarrow A$, and if $\Gamma \vdash_{\mathrm{DF} / \mathrm{TTm}}$ $A \leftrightarrow B$, then also $\Gamma \vdash \mathrm{DF} / \mathrm{TTm} B \leftrightarrow A$. However, transitivity fails, for otherwise $\vdash_{\mathrm{DF} / \mathrm{TTm}}$ would be unsound (consider a DF/TT-evaluation $v$ in which $v(A)=1$, $v(B)=1 / 2$, and $v(C)=0)$.

As the above proof shows, the failure of transitivity for DF/TTm-provable equivalence is closely connected to the failure of Modus Ponens for DF/TT. However, even though $\sim_{\Gamma}^{d f}$ is not an equivalence relation on For $\times$ For, we will see that it is sufficiently well-behaved to support an application of the Lindenbaum-Tarski method. More specifically, due to failure of transitivity, $\sim_{\Gamma}^{\mathrm{df}}$ fails to partition For $\times$ For into 
equivalence classes. Nevertheless, we can still use $\sim_{\Gamma}^{\text {df }}$ to define sets of sets of formulae which still support an application of the Lindenbaum-Tarski method, and therefore a proof of algebraic completeness. This is done in the following definition.

Definition 4.4 For every $\{\Gamma, A\} \subseteq$ For, let $[A]_{\Gamma}^{\text {df }}$ denote the set of formulae that are provably DF/TTm-equivalent to $A$. The quotient induced by $\sim_{\Gamma}^{\text {df }}$ on For, in symbols For $/ \sim_{\Gamma}^{\mathrm{df}}$, is the set of sets in which $\sim_{\Gamma}^{\mathrm{df}}$ subdivides For.

More formally, For $/ \sim_{\Gamma}^{\mathrm{df}}$ is the set of sets $\left\{A \in\right.$ For $\mid \Gamma \vdash_{\mathrm{DF} / T T m} A \leftrightarrow$ $B$ for some $B \in$ For $\}$. Since we are only concerned with de Finetti algebrae in this subsection, we drop the superscript ${ }^{\text {df }}$ and simply write $[A]_{\Gamma}$, in order to improve readability.

Definition 4.5 The de Finetti-Lindenbaum-Tarski algebra of $\Gamma$ is the structure

$$
\mathcal{D}(\Gamma)=\left\langle\text { For } / \sim_{\Gamma}, \Pi_{\Gamma}, \sqcup_{\Gamma},-_{\Gamma}, \nabla_{\Gamma}, \mathbf{0}_{\Gamma}, \mathbf{1} / \mathbf{2}_{\Gamma}, \mathbf{1}_{\Gamma}\right\rangle
$$

where:

$$
\begin{array}{ll}
{[A]_{\Gamma} \sqcap_{\Gamma}[B]_{\Gamma}:=[A \wedge B]_{\Gamma}} & {[A]_{\Gamma} \sqcup_{\Gamma}[B]_{\Gamma}:=[A \vee B]_{\Gamma}} \\
-_{\Gamma}[A]_{\Gamma}:=[\neg A]_{\Gamma} & {[A]_{\Gamma} \vee_{\Gamma}[B]_{\Gamma}:=[A \rightarrow B]_{\Gamma}} \\
{[\perp]_{\Gamma}:=\mathbf{0}_{\Gamma}} & {[\top]_{\Gamma}:=\mathbf{1}_{\Gamma}} \\
& {[\perp \rightarrow \top]_{\Gamma}:=\mathbf{1} / \mathbf{2}_{\Gamma}}
\end{array}
$$

As shown by Lemma $4.3, \sim_{\Gamma}$ is not an equivalence relation, and the sets $[A]_{\Gamma}$ are not equivalence classes. Therefore, there is no guarantee that every formula belongs to exactly one of the elements in For $/ \sim_{\Gamma}$. So, we have to prove that the operations that characterize de Finetti-Lindenbaum-Tarski algebrae, that is $\Pi_{\Gamma}, \sqcup_{\Gamma},-_{\Gamma}$, and $\nabla_{\Gamma}$ are actually well-defined, and do not depend on the choice of particular formulae: otherwise $\Pi_{\Gamma}, \sqcup_{\Gamma},-_{\Gamma}$, and $\nabla_{\Gamma}$ might not be operations at all. This is done in the following lemma.

Lemma 4.6 (Independence from representatives) For every set $\{\Gamma, A, B, C, D\} \subseteq$ For, the following holds:

- If $A \sim_{\Gamma} B$, then $\neg A \sim_{\Gamma} \neg B$.

- If $A \sim_{\Gamma} B$ and $C \sim_{\Gamma} D$, then $(A \wedge C) \sim_{\Gamma}(B \wedge D)$.

- If $A \sim_{\Gamma} B$ and $C \sim_{\Gamma} D$, then $(A \vee C) \sim_{\Gamma}(B \vee D)$.

- If $A \sim_{\Gamma} B$ and $C \sim_{\Gamma} D$, then $(A \rightarrow C) \sim_{\Gamma}(B \rightarrow D)$.

Proof We only show the cases of negation and conditional (the others are similar). For the case of negation, suppose that there is a set $\{\Gamma, A, B\} \subseteq$ For such that $A \sim_{\Gamma} B$ but that it is not the case that $\neg A \sim_{\Gamma} \neg B$. This means that $\Gamma \vdash_{\mathrm{DF} / \mathrm{TTm}} A \leftrightarrow B$ but $\Gamma \nvdash$ DF/TTm $\neg A \leftrightarrow \neg B$. By the completeness of DF/TTm (Proposition 3.6), this means that $\Gamma \models \mathrm{DF} / \mathrm{TT} A \leftrightarrow B$ but $\Gamma \not \nvdash_{\mathrm{DF} / \mathrm{TT}} \neg A \leftrightarrow \neg B$. Let $v$ be any DF-evaluation 
that assigns value 1 or $1 / 2$ to all the sentences in $\Gamma$, value 1 or $1 / 2$ to $A \leftrightarrow B$ but value 0 to $\neg A \leftrightarrow \neg B$ (if there are no DF-evaluations that assign values 1 or $1 / 2$ to all the sentences in $\Gamma$, the claim is immediate). A biconditional is assigned value 0 by a DF-evaluation just in case that evaluation assigns value 1 to one side of the biconditional and 0 to the other. Suppose without loss of generality that $v(\neg A)=1$ and $v(\neg B)=0$. Since $v$ is a DF-evaluation, $v(A)=0$ and $v(B)=1$. But then $v(A \leftrightarrow B)=0$, against our supposition. ${ }^{15}$

For the case of the conditional, suppose that there is a set $\{\Gamma, A, B, C, D\} \subseteq$ For such that $A \sim_{\Gamma} B$ and $C \sim_{\Gamma} D$ but that it is not the case that $(A \rightarrow C) \sim_{\Gamma}(B \rightarrow$ $D)$. This means that $\Gamma \vdash_{\mathrm{DF} / \mathrm{TTm}} A \leftrightarrow B$ and $\Gamma \vdash_{\mathrm{DF} / \mathrm{TTm}} C \leftrightarrow D$ but $\Gamma \nvdash_{\mathrm{DF} / \mathrm{TTm}}$ $(A \rightarrow C) \leftrightarrow(B \rightarrow D)$. Again by the completeness of DF/TTm (Proposition 3.6), this means that $\Gamma \models \mathrm{DF} / \mathrm{TT} A \leftrightarrow B$ and $\Gamma \models_{\mathrm{DF} / \mathrm{TT}} C \leftrightarrow D$ but $\Gamma \nvdash_{\mathrm{DF} / \mathrm{TT}}(A \rightarrow C) \leftrightarrow$ $(B \rightarrow D)$. Let $v$ be any DF-evaluation that assigns value 1 or $1 / 2$ to all the sentences in $\Gamma$, value 1 or $1 / 2$ to $A \leftrightarrow B$ and to $C \leftrightarrow D$ but value 0 to $(A \rightarrow C) \leftrightarrow(B \rightarrow D)$ (if there are no DF-evaluations that assign values 1 or $1 / 2$ to all the sentences in $\Gamma$, the claim is immediate). As noted above, a biconditional is assigned value 0 by a DFevaluation just in case that evaluation assigns value 1 to one side of the biconditional and 0 to the other. Suppose without loss of generality that $v(A \rightarrow C)=1$ and $v(B \rightarrow D)=0$. Since $v$ is a DF-evaluation, $v(A)=v(C)=v(B)=1$ and $v(D)=0$. But then $v(C \leftrightarrow D)=0$, against our supposition.

Lemma 4.7 For every $\Gamma \subseteq$ For, $\mathcal{D}(\Gamma)$ is a de Finetti algebra.

Proof It is easy to see that the properties of distributive bounded lattices hold for $\mathcal{D}(\Gamma)$. We do just one case of distributivity in detail.

$$
\begin{aligned}
{[A]_{\Gamma} \sqcap_{\Gamma}\left([B]_{\Gamma} \sqcup_{\Gamma}[C]_{\Gamma}\right) } & =[A]_{\Gamma} \sqcap_{\Gamma}[B \vee C]_{\Gamma} & & \text { definition of } \mathcal{D}(\Gamma) \\
& =[A \wedge(B \vee C)]_{\Gamma} & & \text { definition of } \mathcal{D}(\Gamma) \\
& =[(A \wedge B) \vee(A \wedge C)]_{\Gamma} & & \text { logic } \\
& =[A \wedge B]_{\Gamma} \sqcup_{\Gamma}[A \vee C]_{\Gamma} & & \text { definition of } \mathcal{D}(\Gamma) \\
& =\left([A]_{\Gamma} \sqcap_{\Gamma}[B]_{\Gamma}\right) \sqcup_{\Gamma}\left([A]_{\Gamma} \sqcap_{\Gamma}[C]_{\Gamma}\right) & & \text { definition of } \mathcal{D}(\Gamma)
\end{aligned}
$$

The line labeled with 'logic' abbreviates the fact that the corresponding identity is proven by the fact that $\Gamma \vdash_{\mathrm{DF} / \mathrm{TTm}}(A \wedge(B \vee C)) \leftrightarrow((A \wedge B) \vee(A \wedge C))$.

As for the involution, suppose that $[A]_{\Gamma} \sqsubseteq_{\Gamma}[B]_{\Gamma}$, where $\bigsqcup_{\Gamma}$ is the partial order induced on For $/ \sim_{\Gamma}^{\text {df }}$ by $\Pi_{\Gamma}$ and $\sqcup_{\Gamma}$. By Definition 4.1, this means that $\left([A]_{\Gamma} \Pi_{\Gamma}\right.$ $\left.[B]_{\Gamma}\right)=[A]_{\Gamma}$. However, $(A \wedge B) \leftrightarrow A$ and $A \rightarrow B$ have the same DF truth table, and are DF/TTm-provably equivalent. Therefore, in particular, $\Gamma \vdash_{\mathrm{DF} / \mathrm{TTm}}((A \wedge B) \leftrightarrow$

\footnotetext{
${ }^{15}$ Notice that the above reasoning breaks down for CC/TT, due to the different truth table for the conditional (and hence the biconditional). More specifically, a biconditional $A \leftrightarrow B$ is assigned value 0 by a $\mathrm{CC}$-evaluation if that evaluation assigns value 1 or $1 / 2$ to one side of the biconditional and 0 to the other. In the case of a CC-evaluation $v$ that assigns value $1 / 2$ to $\neg A$ and 0 to $\neg B$, we have that $v(\neg A \leftrightarrow \neg B)=0$ but $v(A \leftrightarrow B)=1 / 2$. See also Lemma 4.18 below.
} 
$A) \leftrightarrow A \rightarrow B$. It follows that $\sqsubseteq_{\Gamma}$ inherits the features of $\nabla_{\Gamma}$ in $\mathcal{D}(\Gamma)$, and thus the claim is established by contraposition. More precisely: ${ }^{16}$

$$
\begin{aligned}
\left([A]_{\Gamma} \sqcap_{\Gamma}[B]_{\Gamma}\right)=[A]_{\Gamma} & \text { iff }\left([A \wedge B]_{\Gamma}\right)=[A]_{\Gamma} \\
& \text { iff } \Gamma \vdash_{\mathrm{DF} / \mathrm{TTm}}(A \wedge B) \leftrightarrow A \\
& \text { iff } \Gamma \vdash_{\mathrm{DF} / \mathrm{TTm}} A \rightarrow B \\
& \text { iff } \Gamma \vdash_{\mathrm{DF} / \mathrm{TTm}} \neg B \rightarrow \neg A \\
& \text { iff } \Gamma \vdash_{\mathrm{DF} / \mathrm{TTm}}(\neg B \wedge \neg A) \leftrightarrow \neg B \\
& \text { iff }\left([\neg B \wedge \neg A]_{\Gamma}\right)=[\neg B]_{\Gamma} \\
& \text { iff }\left(--_{\Gamma}[B]_{\Gamma} \sqcap_{\Gamma}-{ }_{\Gamma}[A]_{\Gamma}\right)=-_{\Gamma}[B]_{\Gamma}
\end{aligned}
$$

The second constraint on involution is also satisfied, since $\Gamma \vdash_{\mathrm{DF} / \mathrm{TTm}} A \leftrightarrow \neg \neg A$.

The De Morgan and Kleene properties, as well as the defining equation of $\nabla_{\Gamma}$, are proven in a similar way.

We now provide algebraic counterparts of the notions of evaluation and TTconsequence. More precisely, we provide a local notion of algebraic TT-consequence (TT-consequence with respect to a single de Finetti algebra) and a global one (TTconsequence with respect to a class of de Finetti algebrae). This is done in the next two definitions.

Definition 4.8 Let $\mathcal{D}$ be a de Finetti algebra with support $\bar{D}$. A $\mathcal{D}$-evaluation is a function $e:$ For $\longmapsto \bar{D}$ s.t.:

$$
\begin{aligned}
e(\neg A) & :=-e(A) \\
e(A \wedge B) & :=e(A) \sqcap e(B) \\
e(A \vee B) & :=e(A) \sqcup e(B) \\
e(A \rightarrow B) & :=e(A) \triangleright e(B)
\end{aligned}
$$

Definition 4.9 For every class of de Finetti algebrae $\mathfrak{D}$, every de Finetti algebra $\mathcal{D} \in \mathfrak{D}$, and every set $\{\Gamma, A\} \subseteq$ For:

$A$ is a $\mathcal{D}$-consequence of $\Gamma$, in symbols $\Gamma \models \mathcal{D} A$ if for every $\mathcal{D}$-evaluation $e$, if for every $B \in \Gamma, e(B)=\mathbf{1}$ or $\mathbf{1} / \mathbf{2}$, then $e(A)=\mathbf{1}$ or $\mathbf{1} / \mathbf{2}$.

$A$ is a $\mathfrak{D}$-consequence of $\Gamma$, in symbols $\Gamma \models \mathfrak{D} A$, if for every $\mathcal{D} \in \mathfrak{D}, A$ is a $\mathcal{D}$-consequence of $\Gamma$.

Notice that, even though de Finetti algebrae include an algebraic counterpart of the Łukasiewicz trivalent conditional, the latter is not used in defining an algebraic

\footnotetext{
${ }^{16}$ Note that the following reasoning does not rely on uses of Modus Ponens in DF/TTm, but can be carried out using the soundness and completeness of the calculus DF/TTm.
} 
evaluation for de Finetti algebrae (and it is not going to be used to construct specific algebraic models of DF/TT either). The reason behind this choice is that we want to isolate the de Finetti conditional, and the respective TT-logic, without including extraneous connectives (such as the Łukasiewicz conditional). However, it would be possible to expand our definition of algebraic evaluations and algebraic consequence to include the Łukasiewicz trivalent conditional, and prove the relative algebraic soundness and completeness theorems by adding suitable multi-sequent rules to DF/TTm.

We can finally use the structures introduced above, as well as the algebraic notions of consequence, to establish an algebraic soundness and completeness result. This is done in the next two results.

Lemma 4.10 For every set $\{\Gamma, A\} \subseteq$ For:

$$
\Gamma \vdash_{\mathrm{DF} / \mathrm{TTm}} A \text { if and only if } \Gamma \models \mathcal{D}(\Gamma) A
$$

Proof sketch The left-to-right direction is proven by induction on the length of derivations in DF/TTm. As for the right-to-left direction, suppose that $\Gamma \nvdash \mathrm{DF} / \mathrm{TTm} A$. By Proposition 3.4, the sequent $\Gamma|A| A$ has a countermodel, that is, there is a function $v:$ For $\longmapsto\{0,1 / 2,1\}$ s.t. for every $B \in \Gamma, v(B)=1 / 2$ or 1 , but $v(A)=0 .{ }^{17}$ Let $P$ be the set of propositional variables in $\{\Gamma, A\}$. Let $\widetilde{e}$ be the partial function $\widetilde{e}:$ For $\rightarrow$ For $/ \sim_{\Gamma}^{\mathrm{df}}$ defined as follows:

$$
\widetilde{e}\left(p_{i}\right)=\left\{\begin{array}{l}
\mathbf{0}_{\Gamma}, \text { if } p_{i} \in P \text { and } v\left(p_{i}\right)=0 \\
\mathbf{1}_{\Gamma}, \text { if } p_{i} \in P \text { and } v\left(p_{i}\right)=1 / 2 \\
\mathbf{1}_{\Gamma}, \text { if } p_{i} \in P \text { and } v\left(p_{i}\right)=1
\end{array}\right.
$$

By Zorn's Lemma, $\tilde{e}$ can be expanded to a total function $e$ obeying the clauses of Definition 4.8, i.e., a DF-evaluation. ${ }^{18}$ By Lemma 4.7, $e$ is a $\mathcal{D}(\Gamma)$-evaluation, and by construction for every $B \in \Gamma, e(B)=\mathbf{1}_{\Gamma}$ or $\frac{1}{2}{ }_{\Gamma}$, but $e(A)=\mathbf{0}_{\Gamma}$. This shows that $\Gamma \not \mathcal{D}_{(\Gamma)} A$, as desired.

Proposition 4.11 (Algebraic soundness and completeness) Let $\mathfrak{D}$ be the class of all the de Finetti algebrae. For every set $\{\Gamma, A\} \subseteq$ For:

$$
\Gamma \vdash_{\mathrm{DF} / \mathrm{TTm}} A \text { if and only if } \Gamma \models \mathfrak{D} A
$$

\footnotetext{
${ }^{17}$ Observe that the range of $v$ is the usual value space of de Finetti evaluations, that is, the set $\{0,1 / 2,1\}$, not the set of designated elements $\mathbf{0}_{\Gamma}, \mathbf{1} / \mathbf{2}_{\Gamma}$, and $\mathbf{1}_{\Gamma}$ of $\mathcal{D}(\Gamma)$.

${ }^{18}$ More specifically, one first observes that $\widetilde{e}$ is a quasi-DF-evaluation. It is easy to check that the collection of quasi- DF-evaluations and DF-evaluations forms a partially ordered set $P$, induced by the inclusion relation $(\subseteq)$. Moreover, every totally ordered subset $Q$ of $P$ contains an upper bound, that is a quasiDF-evaluation or a DF-evaluation which is not extended by any other element in $Q$. By Zorn's Lemma, then, $P$ itself contains a maximal element, i.e., a quasi-DF-evaluations or a DF-evaluation $e$ s.t. $\widetilde{e} \subseteq e$. It is then immediate to observe than $e$ is a DF-evaluation and not a quasi-DF-evaluation, and hence that $\tilde{e} \varsubsetneqq e$, for otherwise it would be itself expanded by another element in $P$, and therefore it would not be maximal.
} 
Proof sketch The left-to-right direction is straightforward. As for the right-to-left direction, suppose that $\Gamma \nvdash_{\mathrm{DF} / \mathrm{TTm}} A$. By Lemma 4.10, this entails that $\Gamma \nvdash_{\mathcal{D}(\Gamma)} A$,

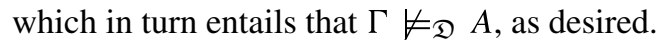

It should be noted that the proof of algebraic completeness just given is not, strictly speaking, a genuine algebraic proof: it is parasitic on the Schütte-style completeness proof given in the previous Section 3.1. ${ }^{19}$ More precisely, the Schütte-style proof is used to construct a countermodel based on the de Finetti algebra with just three elements, $\mathbf{0}, \mathbf{1} / \mathbf{2}$, and $\mathbf{1}$, which is then expanded to an evaluation based on $\mathcal{D}(\Gamma)$. A typical algebraic proof would proceed by establishing a canonical model theorem; however, DF/TT does not seem to support this result. We can explain the specific features of the completeness theorem for DF/TT as follows. ${ }^{20}$

Lemma 4.10 and Proposition 4.11 entail that $\Gamma \models_{\mathcal{D}(\Gamma)} A$ if and only if $\Gamma \models_{\mathfrak{D}} A$. While the right-to-left direction of this biconditional is not surprising, ${ }^{21}$ its left-toright direction yields that $A$ follows from $\Gamma$ in the specific de Finetti algebra $\mathcal{D}(\Gamma)$ just in case $A$ follows from $\Gamma$ in the class of all de Finetti algebrae. That is, the question of whether $A$ is a consequence of $\Gamma$ in all de Finetti algebrae is reduced to the question of whether $A$ is a consequence of $\Gamma$ in $\mathcal{D}(\Gamma)$. However, this appears less surprising if one considers how $\mathcal{D}(\Gamma)$ is constructed: in fact, $\mathcal{D}(\Gamma)$ is built using the very sentences that make up the target inference from $\Gamma$ to $A .^{22}$ Indeed, algebraic completeness proofs typically establish even stronger results, i.e., they establish a canonical model theorem. Such a theorem guarantees that there is exactly one evaluation $e$ (in our case, it would be a de Finetti (algebraic) evaluation) which assigns the designated value to all the elements of $\Gamma$ such that $e$ assigns the designated value to $A$ if and only if $\Gamma \models \mathfrak{D} A$. That is, rather than reducing the question of whether $A$ is a consequence of $\Gamma$ in all algebrae of a certain kind to the question of whether $A$ is a consequence of $\Gamma$ in a single algebra of that kind, a canonical model theorem reduces the former question to the question of whether $A$ has a designated value in a single evaluation. And this is a much stronger result because, in order to establish that $\Gamma \models \mathcal{D}(\Gamma) A$, one has to consider all the de Finetti algebraic evaluations based on $\mathcal{D}(A)$, whereas if a canonical model were available, one would just need to consider one such evaluation (a particularly informative and 'canonical' one). This happens, for example, in proofs of algebraic completeness for classical logic (with respect to the class of all Boolean algebrae), and for several other logics as well. Many of these logics are also algebraizable.

The situation is visualized in Fig. 2: a circle is an algebra, a dot in a circle is an evaluation based on that algebra, and the collection of circles is a class of algebrae. Our completeness result establishes that, for every inference, there is one circle such

\footnotetext{
${ }^{19}$ See the proof of Proposition 3.4.

${ }^{20}$ We thank an anonymous referee for prompting us to comment on this issue.

${ }^{21}$ Lemma 4.7 establishes that $\mathcal{D}(\Gamma)$ is a de Finetti algebra, and hence $\mathcal{D}(\Gamma) \in \mathfrak{D}$, as in Proposition 4.11 we explicitly assume that $\mathfrak{D}$ is the class of all de Finetti algebrae.

${ }^{22}$ There are immediate parallels outside of abstract algebraic logic if one looks at how Henkin models are defined in standard completeness proofs for pure classical logic, or if one considers canonical models in completeness proofs for normal modal logics.
} 

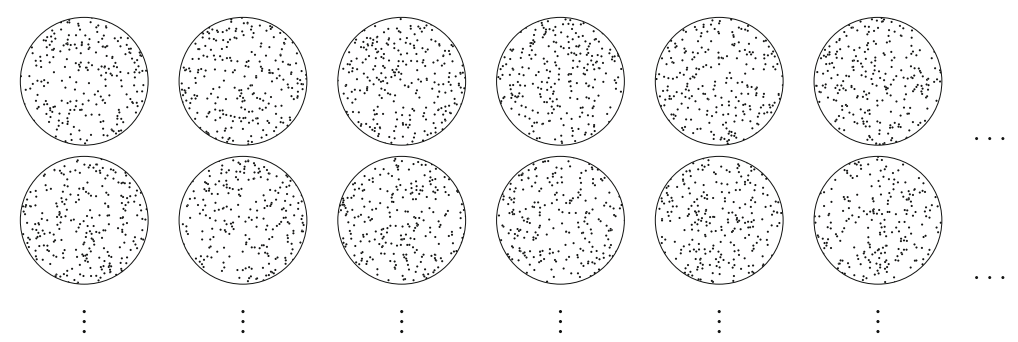

Fig. 2 A graphical representation of the evaluation of inferences in classes of algebrae.

that that inference is valid in all the circles if and only if it is valid in that circle (which in turn requires considering all the dots in that circle). A canonical model theorem establishes a much stronger claim, namely that for every inference there is one single dot in one of the circles such that that inference holds in all the circles if and only if its conclusion receives the designated value in that dot.

Crucially, however, proving a canonical model theorem typically requires Modus Ponens. Indeed, an attempted proof of a canonical model theorem for DF/TT breaks down exactly where Modus Ponens is required. In absence of Modus Ponens, only the weaker result that $\Gamma=_{\mathcal{D}(\Gamma)} A$ if and only if $\Gamma \models_{\mathfrak{D}} A$ holds. However, this is enough for our purposes, that is proving algebraic completeness for DF/TT. In the next subsection, we will see that things do not work so well for CC/TT and the TT-logics of Jeffrey conditionals more generally.

We conclude this subsection with a brief discussion of the prospects for a full algebraizability of DF/TT. ${ }^{23}$ In this respect, however, DF/TT reveals non-negligible limitations. Let's start with some preliminary definitions.

\section{Definition 4.12}

- Let an equation, in symbols $A \approx B$, be a pair of formulae of $\mathcal{L} \rightarrow$. The set of all equations is therefore For $\times$ For. A set of equations, i.e., a subset of For $\times$ For, is denoted by $\mathbb{E}$.

- Let $\mathcal{D}$ be a de Finetti algebra, and let $\{\mathbb{E}, C \approx D\}$ be a set of equations. $\mathcal{D}$ validates the equational inference from $\mathbb{E}$ to $C \approx D$, in symbols $\mathbb{E} \models_{\mathcal{D}} C \approx D$, if for every $\mathcal{D}$-evaluation $e$ if, if for every equation $(A \approx B) \in \mathbb{E}, e(A)=e(B)$, then $e(C)=e(D) .^{24}$

- Let $\mathfrak{D}$ be a class of de Finetti algebrae. $\mathfrak{D}$ validates the equational inference from $\mathbb{E}$ to $C \approx D$, in symbols $\mathbb{E} \models_{\mathfrak{D}} A \approx B$, if for every $\mathcal{D} \in \mathfrak{D}, \mathbb{E} \models \mathcal{D} A \approx B$.

We can now formulate the notion of algebraizability.

\footnotetext{
${ }^{23}$ The notion of algebraizability (introduced by Blok and Pigozzi [8]) generalizes the link between a logic and its algebraic semantics, imposing stricter conditions than those required for algebraic completeness. See also Herrmann [20] and Font [17, Chapters 2 and 3].

${ }^{24}$ Informally, $\mathcal{D}$ validates the equational inference from $\mathbb{E}$ to $C \approx D$ if every de Finetti evaluation based on $\mathcal{D}$ that satisfies all the equations in $\mathbb{E}$, also satisfies $C \approx D$.
} 
Definition 4.13 A logic $L$ is algebraizable if there are a class of algebrae $\mathfrak{A}$ and functions $f: \mathcal{P}$ (For) $\longmapsto \mathcal{P}$ (For $\times$ For) and $g: \mathcal{P}$ (For $\times$ For $) \longmapsto \mathcal{P}$ (For) s.t. for every $\{\Gamma, A\} \subseteq$ For: $^{25}$

(A1) $\quad \Gamma \vdash_{\mathrm{L}} A$ if and only if $f[\Gamma] \models_{\mathfrak{A}} f(A)$

$$
B \approx C=\mid=\mathfrak{A} f(g(B \approx C))
$$

(A1) is a generalization of algebraic completeness, where the right-hand side expresses in the object-language the requirement that $A$ has a designated value whenever all the sentences in $\Gamma$ do. $^{26}$ (A2) ensures that the solvability of equations is fully captured by some formula of the object-language. ${ }^{27}$

However, (A1) and (A2) do not sit well with the conditional of DF/TT. In the context of DF/TT, the right-hand side of (A1) expresses that whenever all the sentences in $\Gamma$ have value 1 or $\frac{1}{2}$, so does $A$. A plausible formalization is obtained by letting $f$ be $f(A)=A \approx(A \leftrightarrow \top) \vee(A \leftrightarrow(\perp \rightarrow \top)) . f[\Gamma]=\mathfrak{D} f(A)$ then becomes

$$
\bigcup_{B \in \Gamma} B \approx(B \leftrightarrow \top) \vee(B \leftrightarrow(\perp \rightarrow \top)) \models \mathfrak{D} A \approx(A \leftrightarrow \top) \vee(A \leftrightarrow(\perp \rightarrow \top)),
$$

Define $g$ as $g(\mathbb{E})=\{B \leftrightarrow C \in$ For $\mid B \approx C \in \mathbb{E}\}$. (A2) becomes

$$
B \approx C=\models_{\mathfrak{D}}(B \leftrightarrow C) \approx((B \leftrightarrow C) \leftrightarrow \top) \vee((B \leftrightarrow C) \leftrightarrow(\perp \rightarrow \top))
$$

which, however, does not express the idea that $B$ and $C$ have the same value whenever $B \leftrightarrow C$ holds in DF/TT. In fact, it is not the case that, in order for $B \leftrightarrow C$ to have the same value as $((B \leftrightarrow C) \leftrightarrow \top) \vee((B \leftrightarrow C), B$ has to have the same value as $C$; an evaluation $e$ such that $e(B)=1$ and $e(C)=1 / 2$ provides a counterexample. This translates into the algebraic semantics, considering a de Finetti algebra $\mathcal{D}$ and an algebraic evaluation $e$ based on $\mathcal{D}$ s.t. $e(B)=\mathbf{1}$ and $e(C)=\frac{1}{2}$ (for $\mathbf{1}, \frac{1}{2} \in \bar{D}$ ).

Of course, this observation only tells us that $B \leftrightarrow C$ does not express the fact that $B$ and $C$ have the same value-which is not surprising, given the 1 - and 1/2-rows of the truth table of the de Finetti conditional. However, the idea of mapping identity of semantic values to a formula that expresses 'having a designated value' seems at odds with the conditional of DF/TT, because DF/TT does not distinguish between 1 and $1 / 2$ when it comes to designatedness, nor does its conditional. In a toleranttolerant semantics, validity (and the corresponding conditionals) does not depend on the identity of the semantic values that are preserved from premises to conclusion, but on their similarity: 1 and $1 / 2$ are not identical, but similar enough for DF/TT

\footnotetext{
${ }^{25}$ Let ' $f[\Gamma]$ ' be a shorthand for $\{f(B) \in \mathcal{P}$ (For $\times$ For) $\mid B \in \Gamma\}$.

${ }^{26}$ For example, consider the case of classical logic and Boolean algebrae. Letting $f$ be the function defined as $f(A)=A \approx \top$, and $\mathfrak{B}$ be the class of Boolean algebrae, $f[\Gamma] \models_{\mathfrak{B}} f(A)$ becomes $\bigcup_{B \in \Gamma} B \approx \top \models_{\mathfrak{B}}$ $A \approx \mathrm{T}$, which formalizes the idea that whenever every sentence in $\Gamma$ has value $\mathbf{1}$, so does $A$.

${ }^{27}$ Consider again classical logic and Boolean algebrae. Classically, $B$ and $C$ have the same value just in case $B \equiv_{m} C$ (where $\equiv_{m}$ denotes the material biconditional) has value 1. Letting $g$ be s.t. $g(\mathbb{E})=\left\{B \equiv_{m}\right.$ $C \in$ For $\mid B \approx C \in \mathbb{E}\}, B \approx C=\models_{\mathfrak{B}} f(g(B \approx C))$ becomes $B \approx C=\models_{\mathfrak{B}}\left(B \equiv_{m} C\right) \approx \top$, which expresses the idea that $B$ and $C$ have the same value whenever the corresponding biconditional holds (i.e. has value 1) in classical logic.
} 
not to distinguish them. But condition (A2) can only be satisfied via a formula that captures a notion of validity based on the identity of semantic values.

This does not show that DF/TT is not algebraizable: here, we leave the question open. However, the above observations suggest a possible strategy to prove nonalgebraizability: if one can show that a truth-function expressing identity of truth values is not definable in the truth table semantics for DF, this would translate into the algebraic semantics, and establish non-algebraizability. ${ }^{28}$

\subsection{An algebraic Semantics for CC/TT?}

Can we provide a proof of algebraic completeness for CC/TT employing the Lindenbaum-Tarski method, as we did for DF/TT? The CC/TT-conditional appears better-behaved than the DF/TT one-in particular because it obeys Modus Ponensso this would appear prima facie possible.

Let us try to apply the Lindenbaum-Tarski method to CC/TT. First, we need an algebraic counterpart of the Cooper-Cantwell conditional. This is provided by the following definition.

Definition 4.14 An $Ł 3$ algebra $\mathcal{A}=\langle\bar{A}, \sqcap, \sqcup,-, \longmapsto, \triangleright, \mathbf{0}, \mathbf{1}, \mathbf{1} / 2\rangle$ is CooperCantwell if:

There is a distinguished element $\mathbf{1} / \mathbf{2} \in \bar{A}$ s.t. $-\mathbf{1} / \mathbf{2}=\mathbf{1} / \mathbf{2}$, and

There is an operation $\triangleright$ defined on $\bar{A} \times \bar{A}$ s.t. $a \triangleright b=-\mathrm{w}(a) \sqcup(\mathrm{w}(a) \sqcap b)$, where $\mathrm{w}(a)$ is a shorthand for $-a \longmapsto \mathbf{1 / 2}$.

We then work towards the construction of a Lindenbaum-Tarski algebra for CC/TT.

Definition 4.15 For every $\Gamma \subseteq$ For, let $\sim_{\Gamma}^{c} \subseteq$ For $\times$ For be the relation defined as follows:

$$
A \sim_{\Gamma}^{c} B \text { if and only if } \Gamma \vdash_{\mathrm{CC} / \mathrm{TTm}} A \leftrightarrow B
$$

Since Modus Ponens holds in CC/TT, the relation of CC/TTm-provable equivalence seems better behaved than the one defined for DF/TTm.

Lemma 4.16 $\sim_{\Gamma}^{c}$ is an equivalence relation on For $\times$ For.

Proof Reflexivity holds since $\Gamma \vdash_{\mathrm{CC} / \mathrm{TTm}} A \leftrightarrow A$. Symmetry also holds, since if $\Gamma \vdash_{\mathrm{cC} / \mathrm{TTm}} A \leftrightarrow B$, then also $\Gamma \vdash_{\mathrm{cC} / \mathrm{TTm}} B \leftrightarrow A$. Finally, transitivity holds as well,

\footnotetext{
${ }^{28}$ This proof strategy seems simpler and more informative than a proof via Isomorphism Theorems, which are the standard results employed to prove non-algebraizability (see [17]). Another open question is whether DF/TT is algebraizable over other logics. A natural choice would be Łukasiewicz's trivalent logic (with a TT-notion of validity), because de Finetti algebrae are defined over $Ł 3$ algebrae. There are reasons to expect a positive result, namely that the Łukasiewicz trivalent conditional can be used to express the identity of semantic values, e.g. via $\neg((A \leftrightarrow B) \rightarrow \neg(A \leftrightarrow B))$.
} 
because if $\Gamma \vdash_{\mathrm{cc} / \mathrm{TTm}} A \leftrightarrow B$, and $\Gamma \vdash_{\mathrm{cC} / \mathrm{TTm}} B \leftrightarrow C$ then $\Gamma \vdash_{\mathrm{cC} / \mathrm{TTm}} A \leftrightarrow C$ as well. These equivalences are quickly established semantically, i.e., considering $\models \mathrm{CC} / \mathrm{TTm}$ rather than $\vdash_{\mathrm{CC} / \mathrm{TTm}}$, by the completeness of CC/TTm.

We now have an equivalence relation, so we can use it to partition the set of formulae into equivalence classes.

Definition 4.17 For every $\{\Gamma, A\} \subseteq$ For, let $[A]_{\Gamma}^{\mathrm{C}}$ denote the equivalence class of $A$ induced by $\sim_{\Gamma}^{c}$. The quotient induced by $\sim_{\Gamma}^{c}$ on For, in symbols For $/ \sim_{\Gamma}^{c}$, is the set of equivalence classes induced by $\sim_{\Gamma}^{c}$.

Since we only work with Cooper-Cantwell algebrae in this subsection, we drop the superscript ${ }^{\mathrm{CC}}$ again to improve readability, without risks of confusion. Now, in order to proceed with the proof of algebraic completeness, we would have to define a Cooper-Cantwell version of a Lindenbaum-Tarski algebra. Such a structure would look as follows:

$$
\mathcal{C}(\Gamma)=\left\langle\text { For } / \sim_{\Gamma}^{\mathrm{c}}, \Pi_{\Gamma}, \sqcup_{\Gamma},-_{\Gamma}, \triangleright_{\Gamma}, \mathbf{0}_{\Gamma}, \mathbf{1}_{\Gamma}\right\rangle
$$

where:

$$
\begin{array}{ll}
{[A]_{\Gamma} \Pi_{\Gamma}[B]_{\Gamma}:=[A \wedge B]_{\Gamma}} & {[A]_{\Gamma} \sqcup_{\Gamma}[B]_{\Gamma}:=[A \vee B]_{\Gamma}} \\
-_{\Gamma}[A]_{\Gamma}:=[\neg A]_{\Gamma} & {[A]_{\Gamma} \triangleright_{\Gamma}[B]_{\Gamma}:=[A \rightarrow B]_{\Gamma}} \\
{[\perp]_{\Gamma}:=\mathbf{0}_{\Gamma}} & {[\top]_{\Gamma}:=\mathbf{1}_{\Gamma}} \\
& {[\perp \rightarrow \top]_{\Gamma}:=\mathbf{1} / \mathbf{2}_{\Gamma}}
\end{array}
$$

However, the construction is blocked, because some of its defining operations turn out to be not well-defined. In particular, the Cooper-Cantwell conditional is not substitutive with respect to negation.

Lemma 4.18 There are sets $\{\Gamma, A, B\} \subseteq$ For s.t.

$$
A \sim_{\Gamma} B \text { but it is not the case that } \neg A \sim_{\Gamma} \neg B
$$

Proof It is sufficient to set $\Gamma=\varnothing, A=\top$, and $B=\perp \rightarrow \top$.

This lemma shows that the process of providing an algebraic semantics (via the standard Lindenbaum-Tarski method) for CC/TT stops here: it does not even get off the ground.

In fact, this negative result is more general: it applies to every Jeffrey conditional. Recall that Jeffrey conditionals are required to obey the condition that $f_{\rightarrow}(1,0)=f_{\rightarrow}(1 / 2,0)=0$. Now, the above proof employs exactly the cases in which a conditional has an antecedent with value 1 and a consequent with value 0 , and an antecedent with value 1 and a consequent with value $1 / 2$. Therefore, no Jeffrey conditional is substitutive with respect to negation-under a TT-notion of validity, and a Strong Kleene interpretation of conjunction and negation. In turn, this 
means that no ' J-Lindenbaum-Tarski algebra', where ' $\mathrm{J}$ ' is any Jeffrey conditional, is well-defined, and therefore that no algebraic semantics (via the Lindenbaum-Tarski method) is available for any TT-logic of a Jeffrey conditional.

\section{General Discussion}

This two-part paper has reviewed the main motivations for a trivalent semantics for indicative conditionals, interpreting them as conditional assertions, and defining their truth conditions in analogy with the conditions that settle the winner of a conditional bet (i.e., the bet or assertion is declared void when the antecedent is false). Although the idea goes back to de Finetti [13], and Reichenbach [36, 37], there have been few explorations of the logics induced by the adoption of that semantic scheme. Beside expounding the historical roots of trivalent semantics for conditionals, our paper has given a systematic survey of the different logics that emerge by (i) choosing a truth table for the conditional operator in agreement with the above rationale, and (ii) determining a specific notion of validity (one vs. two designated truth values, pure vs. mixed consequence relations).

As reviewed in Part I, the trivalent approach yields a fully truth-functional semantics with attractive logical and inferential properties. It also provides the conceptual foundations for a probabilistic theory of assertability and reasoning with conditionals along the lines of Adams [1]. For simple conditionals, combining our semantics with defining the assertability of a sentence $A$ as the conditional probability that A is true, given that it has a classical truth value, immediately yields Adams' Thesis that Ast $(A \rightarrow C)=p(C \mid A)$. This property highlights the potential of the trivalent approach for guiding an account of the epistemology of conditionals, and explaining how people reason with them (e.g., [5, 6]). While the semantics of the trivalent conditional is factual - that is, its truth value is a function of matters in the actual world-no such limits are imposed on the scope of the probability functions in judgments of assertability (e.g., $A$ can be practically unverifiable, but the conditional may still be highly assertable).

With respect to the above challenges (i) and (ii), it quickly transpires that any alternative to a tolerant-to-tolerant (TT-) notion of validity would be either too strong (in the sense of licensing undesirable inferences such as implying the converse condtional) or too weak (in the sense of violating the Identity Law $A \rightarrow A$ and not having sentential validities). Only the Cooper-Cantwell conditional, where indeterminate antecedents are exactly treated like true ones, satisfies both the full Deduction Theorem and commutation with negation. For conceptual, empirical and logical reasons (the conditional is essentially interpreted as making an assertion upon supposing the antecedent), these are eminently reasonable properties, apparently favoring CC/TT as the best trivalent logic of the indicative conditional.

The results of Part II nuance this judgment. For both DF/TT and CC/TT we can develop sound and complete calculi based on tableaux (Section 2) and three-sided sequents (Section 3). The latter calculi have the advantage of being simpler and more direct: unlike tableau calculi, they do not establish that an inference is valid by showing that it is impossible to assign a designated value to the premises and an 
undesignated value to the conclusion. Moreover, many-sided sequent calculi make it easier to handle inferences with multiple conclusions, as well as inferences involving infinite sets of sentences.

As soon as we consider the algebraic semantics, however, differences between DF/TT and CC/TT emerge. While provable equivalence fails to be transitive and therefore induces no equivalence relation for DF/TT (Lemma 4.3), we can still use this relation to define a Lindenbaum-Tarski algebra and to show an algebraic soundness and completeness theorem (Proposition 4.11). In other words, $A$ can be derived from $\Gamma$ using one of the above calculi (e.g., many-sided sequents) if and only if a consequence relation holds between $\Gamma$ and $A$ in the associated de Finetti algebrae. The failure of Modus Ponens for DF/TT, however, blocks the construction of a canonical algebraic model.

Things look bleak, by contrast, for CC/TT and other TT-logics based on a Jeffrey conditional. While provable equivalence induces an equivalence relation for these logics, the construction of a Lindenbaum-Tarski algebra does not get off the ground because provable equivalence fails to be substitutive under negation. More precisely, the Cooper-Cantwell biconditional $\leftrightarrow$ falls short of expressing CC/TT-equivalence since $A \leftrightarrow B \not=\mathrm{CC} / \mathrm{TT} \rightarrow A \leftrightarrow \neg B$. Which means that there is not, and cannot be, a fruitful standard algebraic treatment of Jeffrey conditionals. In fact, this is grounded in a defining property of Jeffrey conditionals: to preserve Modus Ponens and to yield a full Deduction Theorem, a trivalent conditional based on the "defective" truth table needs to obey $f_{\rightarrow}(1,0)=f_{\rightarrow}(1 / 2,0)=0$. It is exactly this property which makes substitution under negation fail (Lemma 4.18), and prevents a proper algebraic semantics for Jeffrey conditionals.

Clearly, the failure of substitution under negation is closely related to the failure of contraposition in Jeffrey conditionals - an inference that does not fail in DF/TT. Indeed, the same evaluation provides the counterexamples employed in proving both Proposition 5.6 (Part I) and Lemma 4.18 (Part II). So it turns out that what has been a strength of Jeffrey tolerant-tolerant logics, and CC/TT in particular, at the level of desirable conditional principles, comes at the price of the algebraic semantics. Importantly, the lack of an algebraic semantics is not a mere technical fact, but it has philosophical consequences as well. In particular, in every Jeffrey tolerant-tolerant logic, even if it is the case that $A \leftrightarrow B$, the same equivalence does not hold in general for logically complex sentences that result by uniform substitutions of $A$ and $B$ (see Lemma 4.6 for a formally precise version of this property). Therefore, Jeffrey conditionals do not provide a workable notion of equivalence.

Of course, the limitations of Jeffrey conditionals just reviewed arise from the combination of the semantics of Jeffrey conditionals, TT-validity, and Strong Kleene conjunction, disjunction, and negation: one might therefore wonder whether they can be improved on by altering some of these parameters. However, as the results of Part I show, adopting an alternative to TT-validity does not seem promising. As for the semantics of the other connectives, in part I we noted that Cooper adopted alternative truth tables for conjunction and disjunction, while retaining the K3 table for negation (see also [9, 14] and [21, §7.19, 1044 and following]). This modification gives up some classical properties, such as the inference from $A$ to $A \vee B($ set $v(A)=1 / 2$ and $v(B)=0)$. With regard to the algebraic semantics, 
the difficulties raised in the previous section may not easily be overcome as a result. ${ }^{29}$

Alternatively, one might inquire into what happens to the interaction of Jeffrey conditionals with a non-K3 negation. To be sure, the K3-negation squares particularly well with the philosophical motivation for de Finettian conditionals: when a conditional assertion $A \rightarrow C$ is "called off" because $A$ is false, the same should happen for the negation of that assertion (i.e., the sentence $A \rightarrow \neg C$, thanks to the commutation scheme). Nonetheless, it might be worth investigating how Jeffrey logics (keeping a tolerant-to-tolerant notion of validity) fare when coupled with what [11] call a "Gentzen-regular" negation, that is a negation obeying the Gentzen sequent calculus rules. While a Gentzen-regular negation might avoid some of the above problems, it would lose the commutation of conditional and negation, and the attached connexive principles (see Subsections 5.2 and 5.3 of Part I). In conclusion, there seem to be structural limitations, or at least unavoidable tradeoffs, that affect Jeffrey conditionals, when it comes to their interaction with other connectives.

We therefore believe that it is not easy to justify a clear preference between the two logics CC/TT and DF/TT that we have isolated as most promising amongst trivalent logics of indicative conditionals. Both have attractive properties, both have limitations - but they agree in essential properties such as the valuation of classical sentences, the Import-Export principle, their connexive nature, and the connection to a theory of assertability. To solve the limitations highlighted throughout the paper, one would probably have to give up one or more of these features. So while there is perhaps no perfect trivalent semantics for indicative conditionals, they need to be considered carefully between two-valued truth-functional logic and modal logics of conditionals. In any event, they give rise to a promising research program, and we shall support this claim by sketching some future projects that build on our work in this paper.

First, we would like to extend the current framework to predicate logic and to investigate how the trivalent conditionals fare in that context, including how they interact with a naïve or a compositional truth predicate. Second, we would like to apply trivalent semantics to McGee's famous challenge to Modus Ponens, applying our accounts of logical consequence and probabilistic assertability [29, 40]. Third,

\footnotetext{
${ }^{29}$ A similar application of the Lindenbaum-Tarski method would also likely fail if one replaces strong Kleene conjunction and disjunction with Cooper's quasi-conjunction and quasi-disjunction (see Part I, Section 6), because it only depends on features of the conditional and negation (see Lemma 4.18). However, we don't have proper algebraic counterparts of Cooper's connectives, therefore we can only advance this claim as a conjecture. In particular, some features of Cooper's connectives show that they cannot receive their standard algebraic interpretation. Consider the failure of quasi-disjunction introduction. Standardly, a disjunction is interpreted as the maximum of the two disjuncts, in some order. In a bounded lattice, this means that if an element $a$ has a position with respect to the top element, in the order induced by the lattice, then for every element $b, \max (a, b)$ has a position which is at least as close to the top element as the position of $a$. But this feature seemingly has to fail for an algebraic representation of Cooper's quasi-disjunction, for it corresponds to disjunction introduction. The failure of negated quasi-conjunction introduction (i.e., that the inference from $\neg A$ to $\neg(A \wedge B)$ is not TT-valid for Cooper's quasi-conjunction) seems to have even more far-reaching consequences, for it also involves the involution defined on the corresponding lattices.
} 
one should review the intuitions and inference schemes which fuel connexive logics (e.g., Aristotle's Thesis, Boethius' Thesis) from a trivalent perspective, and conduct a more detailed comparison. Fourth, we can use our theory to respond to recent attacks on Import-Export (e.g., [28]) from a trivalent perspective.

Finally, there is the question of how a trivalent semantics integrates into a general theory of conditionals, including those in the subjunctive mood. Extending a de Finettian treatment of indicative conditionals yields the consequence that all conditionals with false antecedents - in particular, all counterfactuals - have indeterminate truth value. The difference between them is only a difference in assertability (because their conditional probabilities $\operatorname{Pr}(C \mid A)$ can be different and will typically vary with context). This perspective is close to Jeffrey's view which qualifies counterfactual questions either as "nonsense" or as "colorful ways of asking about conditional probabilities" $[23,164]$. On this picture, the traditional view that indicative conditionals are epistemic and counterfactuals are metaphysical [15, 24-26] would be reversed: while indicatives are factual statements (i.e., conditional assertions) with non-trivial truth conditions, counterfactuals come out as having trivial truth conditions and differ only in their epistemic import, that is, their assertability conditions. Whether the proponent of a trivalent semantics for indicative conditionals should be committed to such far-reaching philosophical consequences is, of course, a question that we have to postpone to future research.

Funding Open Access funding enabled and organized by Projekt DEAL.

Open Access This article is licensed under a Creative Commons Attribution 4.0 International License, which permits use, sharing, adaptation, distribution and reproduction in any medium or format, as long as you give appropriate credit to the original author(s) and the source, provide a link to the Creative Commons licence, and indicate if changes were made. The images or other third party material in this article are included in the article's Creative Commons licence, unless indicated otherwise in a credit line to the material. If material is not included in the article's Creative Commons licence and your intended use is not permitted by statutory regulation or exceeds the permitted use, you will need to obtain permission directly from the copyright holder. To view a copy of this licence, visit http://creativecommonshorg/licenses/by/4.0/.

\section{References}

1. Adams, E.W. (1975). The logic of conditionals. Dordrecht: Reidel.

2. Asenjo, F.G. (1966). A calculus of antinomies. Notre Dame Journal of Formal Logic, 16, 103-105.

3. Baaz, M., Fermüller, C., Zach, R. (1992). Dual systems of sequents and tableaux for many-valued logics. Technical Report TUW-E185.2-BFZ.2-92.

4. Baaz, M., Fermüller, C., Zach, R. (1993). Systematic construction of natural deduction systems for many-valued logics: Extended report. Technical Report TUW- E185.2-BFZ.1-93.

5. Baratgin, J., Over, D., Politzer, G. (2013). Uncertainty and the de Finetti tables. Thinking \& Reasoning, 19, 308-328.

6. Baratgin, J., Politzer, G., Over, D.E., Takahashi, T. (2018). The psychology of uncertainty and threevalued truth tables. Frontiers in Psychology, 9, 1479.

7. Beall, J.C. (2009). Spandrels of Truth. Oxford: Oxford University Press.

8. Blok, W.J., \& Pigozzi, D. (1989). Algebraizable Logics. Volume 77 of Memoirs of the American Mathematical Society. American Mathematical Society.

9. Calabrese, P. (2002). Deduction with uncertain conditionals. Information Sciences, 147, 143-191. 
10. Cantwell, J. (2008). The logic of conditional negation. Notre Dame Journal of Formal Logic, 49, 245-260.

11. Chemla, E., \& Égré, P. (2019). From many-valued consequence to many-valued connectives. Synthese. https://doi.org/10.1007/s11229-019-02344-0.

12. Cooper, W.S. (1968). The propositional logic of ordinary discourse. Inquiry, 11, 295-320.

13. de Finetti, B. (1936). La logique de la probabilité. In Actes du congrès international de philosophie scientifique, (Vol. 4 pp. 1-9): Hermann Editeurs Paris.

14. Dubois, D., \& Prade, H. (1994). Conditional objects as nonmonotonic consequence relationships. IEEE Transactions on Systems, Man, and Cybernetics, 24(12), 1724-1740.

15. Edgington, D. (1995). On Conditionals. Mind, 104, 235-329.

16. Farrell, R.J. (1979). Material implication, confirmation, and counterfactuals. Notre Dame Journal of Formal Logic, 20, 383-394.

17. Font, J.M. (2016). Abstract algebraic logic-an introductory textbook. Studies in Logic: Mathematical Logic and Foundations. London: College Publications.

18. Goodship, L. (1996). On dialetheism. Australasian Journal of Philosophy, 74, 153-61.

19. Gottwald, S. (2001). A treatise on many-valued logics. Volume IX of Studies in Logic and Computation. Baldock: Research Studies Press Ltd.

20. Herrmann, B. (1996). Equivalential and algebraizable logics. Studia Logica, 57, 419-436.

21. Humberstone, L. (2011). The connectives. Cambridge: MIT Press.

22. Jeffrey, R.C. (1963). On indeterminate conditionals. Philosophical Studies, 14, 37-43.

23. Jeffrey, R.C. (1991). Matter-of-Fact Conditionals. Proceedings of the Aristotelian Society, 65, 161-183.

24. Khoo, J. (2015). On indicative and subjunctive conditionals. Philosopher's Imprint, 15.

25. Lewis, D. (1973). Causation. Journal of Philosophy, 70, 556-567.

26. Lewis, D. (1973). Counterfactuals. Oxford: Basil Blackwell.

27. Malinowski, G. (1993). Many-valued logics. Oxford: Clarendon Press.

28. Mandelkern, M. (2020). Import-Export and 'And'. Philosophy and Phenomenological Research, $100(1), 118-135$.

29. McGee, V. (1985). A counterexample to modus ponens. The Journal of Philosophy, 82, 462-71.

30. Milne, P. (2004). Algebras of intervals and a logic of conditional assertions. Journal of Philosophical Logic, 33, 497-548.

31. Moschovakis, Y. (1974). Elementary induction on abstract structures. Amsterdam, London and New York: North-Holland and Elsevier.

32. Pohlers, W. (2009). Proof theory. The first step into impredicativity. Berlin: Springer.

33. Priest, G. (1979). The logic of paradox. Journal of Philosophical Logic, 8, 219-241.

34. Priest, G. (2006). Doubt truth to be a liar. Oxford: Oxford University Press.

35. Pynko, A. (1995). On Priest's logic of paradox. Journal of Applied Non-Classical Logics, 5, 219-225.

36. Reichenbach, H. (1935). Wahrscheinlichkeitslehre. Leiden: Sijthoff.

37. Reichenbach, H. (1944). Philosophic foundations of quantum mechanics. Berkeley: University of California Press.

38. Ripley, D. (2012). Conservatively extending classical logic with transparent truth. Review of Symbolic Logic, 5, 354-78.

39. Schütte, K. (1956). Ein System des verknüpfenden Schließens. Archiv für mathematische Logik und Grundlagenforschung, 2, 55-67.

40. Stern, R., \& Hartmann, S. (2018). Two sides of modus ponens. The Journal of Philosophy, 115(11), 605-621.

41. Troelstra, A.S., \& Schwichtenberg, H. (2000). Basic proof theory. Cambridge: Cambridge University Press.

Publisher's Note Springer Nature remains neutral with regard to jurisdictional claims in published maps and institutional affiliations. 


\section{Affiliations}

\section{Paul Égré1 ${ }^{(10}$ . Lorenzo Rossi ${ }^{2}$ (I) . Jan Sprenger ${ }^{3}$ (1)}

1 Institut Jean-Nicod (CNRS/ENS/EHESS), Département de Philosophie \& Département d'études Cognitives, Ecole Normale Supérieure, PSL University, 29 rue d’Ulm, 75005, Paris, France

2 Munich Center for Mathematical Philosophy (MCMP), Fakultät für Philosophie, Wissenschaftstheorie und Religionswissenschaft, Ludwig-Maximilians-Universität München, Geschwister-Scholl-Platz 1, D-80539 München, Germany

3 Center for Logic, Language and Cognition (LLC), Department of Philosophy and Education Science, Università degli Studi di Torino, Via Sant'Ottavio 20, 10124 Torino, Italy 